\title{
OPTIMAL MASS TRANSPORT ON METRIC GRAPHS
}

\author{
J. M. MAZON, J. D. ROSSI AND J. TOLEDO
}

\begin{abstract}
We study an optimal mass transport problem between two equal masses on a metric graph where the cost is given by the distance in the graph. To solve this problem we find a Kantorovich potential as the limit of $p$-Laplacian type problems in the graph where at the vertices we impose zero total flux boundary conditions. In addition, the approximation procedure allows us to find a transport density that encodes how much mass has to be transported through a given point in the graph, and also provide a simple formula of convex optimization for the total cost.
\end{abstract}

November 12, 2014

\section{INTRODUCTION}

A quantum graph is a metric graph in which we associate a differential law with each edge that models the interaction between the two vertices (we denote by $\mathrm{v}$ the vertices in what follows) defining each edge (that we denote by e). The use of quantum graphs (in contrast to more elementary graph models, such as simple unweighted or weighted graphs) opens up the possibility of modeling the interactions between agents identified by the graph's vertices in a far more detailed manner than with standard graphs. Quantum graphs are now widely used in physics, chemistry and engineering (nanotechnology) problems, but can also be used, in principle, in the analysis of complex phenomena taking place on large complex networks, including social and biological networks. Such graphs are characterized by highly skewed degree distributions, small diameter and high clustering coefficients, and they have topological and spectral properties that are quite different from those of the highly regular graphs, or lattices arising in physics and chemistry applications. Quantum graphs are also used to model thin tubular structures, so-called graph-like spaces, they are their natural limits, when the radius of a graph-like space tends to zero. On both, the graph-like spaces and the metric graph, we can naturally define Laplace-like differential operators, $[2,3,10,15]$.

In this paper we are interested in the Monge-Kantorovich mass transport problem on metric graphs. That is, we want to transport a certain amount of material in the graph to a prescribed final distribution minimizing a cost given by the distance inside the graph. Our approach to this problem is based on an idea by Evans and Gangbo, [7], that approximates a Kantorovich potential for a transport problem in the Euclidean space with cost given by the Euclidean distance using the limit as $p$ goes to infinity of a family of $p$-Laplacian type problems. This limit procedure turns out to be quite flexible and allowed us to deal with different transport problems (like optimal matching problems, problems with taxes, etc) in which the cost

Key words and phrases. $p$-Laplacian, metric graphs, optimal transport, convex optimization.

J. M. Mazon and J. Toledo are partially supported by MTM2012-31103 (Spain) and J. D. Rossi by MTM2011-27998 (Spain). 
is given by the Euclidean distance or variants of it. See $[8,11,12,13,14]$. Here we apply these ideas to the optimal transport problem on a metric graph showing again that this approximation procedure is quite powerful since it provides all the relevant information for the transport problem.

To put our optimal mass transport in modern mathematical terms we have to introduce some notation. Let $\Gamma$ be a metric graph (see Section 2 for a precise definition) and consider two nonnegative measures on the graph $\mu$ and $\nu$ with the same total mass, that is,

$$
\int_{\Gamma} \mu=\int_{\Gamma} \nu
$$

The associated optimal transport problem (in its relaxed version, also known as Monge-Kantorovich mass transport problem) reads as follows: find an optimal transport plan, that is, a measure $\gamma(x, y)$ that solves the minimization problem

$$
\min _{\gamma \in \Pi(\mu, \nu)} \int_{\Gamma} \int_{\Gamma} d_{\Gamma}(x, y) d \gamma(x, y)
$$

where $d_{\Gamma}(\cdot, \cdot)$ is the distance in the metric graph and $\Pi(\mu, \nu)$ is the set of measures that have marginals $\mu$ and $\nu$ in the first and second variable respectively. A simple argument using a minimizing sequence shows that there exists an optimal transport plan $\gamma^{*}$.

This minimization problem has a dual formulation: to find a Kantorovich potential $u$, that is, a function that solves the maximization problem

$$
\max _{u \in K_{d_{\Gamma}}(\Gamma)} \int_{\Gamma} u d \eta
$$

where

$$
\eta=\mu-\nu
$$

and $K_{d_{\Gamma}}(\Gamma)$ is the set of 1 -Lipschitz functions on $\Gamma$, that is, functions $u: \Gamma \mapsto \mathbb{R}$ such that $|u(x)-u(y)| \leq d_{\Gamma}(x, y)$ for every $x, y \in \Gamma$.

We can find a Kantorovich potential by an approximation procedure using a sequence of solutions $u_{p}$ to $p$-Laplacian type problems and taking the limit as $p \rightarrow \infty$.

Let us consider solutions to the variational problem

$$
\min _{u \in S_{p}} \frac{1}{p} \int_{\Gamma}\left|u^{\prime}\right|^{p}-\int_{\Gamma} u d \eta
$$

where

$$
S_{p}:=\left\{u \in W^{1, p}(\Gamma): \int_{\Gamma} u=0\right\} .
$$

Such minimizers $u_{p}$ are, in fact, weak solutions to (see the notation afterwards):

$$
\left\{\begin{array}{l}
-\left(\left|u^{\prime}\right|^{p-2} u^{\prime}\right)^{\prime}=\eta \quad \text { on edges, } \\
\sum_{\mathrm{e} \in \mathrm{E}_{\mathrm{v}}(\Gamma)}\left|\frac{\partial u}{\partial x_{\mathrm{e}}}\right|^{p-2} \frac{\partial u}{\partial x_{\mathrm{e}}}(\mathrm{v})=0 \quad \text { on vertices. }
\end{array}\right.
$$

Our first result reads as follows:

Theorem 1.1. There exists a subsequence $p_{j} \rightarrow \infty$ such that

$$
u_{p_{j}} \rightrightarrows u_{\infty}
$$


uniformly in $\bar{\Gamma}$. The limit $u_{\infty} \in K_{d}$ is a Kantorovich potential for the optimal mass transport problem of $\mu$ and $\nu$.

Next, we observe that our approximation procedure gives much more, it allows us to construct a transport density and to provide simple formulas for the total cost. These are the contents of our second result. See Section 2 for notation. Let us write $\eta$ as a measure supported on the edges, denoted by e, plus a sum of deltas supported on the vertices, denoted by $\mathrm{v}$, that is,

$$
\eta=\mu+\sum_{\mathrm{v} \in \mathrm{V}(\Gamma)} a_{\mathrm{v}} \delta_{\mathrm{v}},
$$

where where $a_{\mathrm{v}} \in \mathbb{R}$ and $\mu$ is a Radon measure on $\Gamma$ of the form

$$
\langle\mu, \varphi\rangle=\sum_{\mathrm{e} \in \mathrm{E}(\Gamma)} \int_{0}^{\ell \mathrm{e}}[\varphi]_{\mathrm{e}} d \mu_{\mathrm{e}} \quad \text { for } \varphi \in C(\Gamma),
$$

with $\mu_{\mathrm{e}}$ a Radon mesasures in $\left(0, \ell_{\mathrm{e}}\right)$. Assume the mass balance, $\int_{\Gamma} \eta=0$, is satisfied. This gives,

$$
\int_{\Gamma} d \mu+\sum_{\mathrm{v} \in \mathrm{V}(\Gamma)} a_{\mathrm{v}}=0
$$

Theorem 1.2. Along a subsequence $p_{j} \rightarrow \infty$ we have that

$$
\left|\left[u_{p}\right]^{\prime}(x)\right|^{p-2} \rightarrow \mathbf{a}(x) \quad \forall x \in \Gamma .
$$

This limit function $\mathbf{a} \in L^{\infty}(\Gamma)$, is a transport density for our problem and verifies $\left|\left[u_{\infty}\right]^{\prime}\right|=1$ in $\{\mathbf{a}>0\}$.

Moreover, we obtain the following simple formula of convex optimization:

$$
W_{\mu, a_{\mathrm{v}}}=\min \left\{\sum_{\mathrm{e} \in \mathrm{E}(\Gamma)} \int_{0}^{\ell \mathrm{e}}\left|a_{\mathrm{e}, \mathrm{i}_{\mathrm{e}}}+\int_{0}^{x} d \mu_{\mathrm{e}}(y)\right| d x:\left\{a_{\mathrm{e}, \mathrm{v}}\right\} \text { solve }\left(L_{\mu, a_{\mathrm{v}}}\right)\right\}
$$

for the total cost, $W_{\mu, a_{\mathrm{v}}}$, of the optimal transport problem. Here $\left(L_{\mu, a_{\mathrm{v}}}\right)$ stands for the following system of linear equations for unknowns $a_{\mathrm{e}, \mathrm{v}}$,

$$
\left(L_{\mu, a_{\mathrm{v}}}\right)\left\{\begin{array}{l}
\sum_{\mathrm{v} \in \mathrm{e}} a_{\mathrm{e}, \mathrm{v}}=-\int_{0}^{\ell_{\mathrm{e}}} d \mu_{\mathrm{e}} \quad \text { for every edge } \mathrm{e}, \\
\sum_{\mathrm{e} \in \mathrm{E}_{\mathrm{v}}(\Gamma)} a_{\mathrm{e}, \mathrm{v}}=a_{\mathrm{v}} \quad \text { at every vertex } \mathrm{v} .
\end{array}\right.
$$

Remark 1.3. There exists a large amount of literature dealing with optimal transport problems in networks, see for instance the two excellent Lecture Notes [4] and [5]. We want to point out that the problems studied in these monographs are different to the one we face here. In fact, in those references the authors try to find an optimal network that minimize some energy functional associated with the network. In contrast, here the network is given and our aim is to describe the Kantorovich potential and the transport density of a Monge-Kantorovich mass transport problem on the graph represented by the network.

The paper is organized as follows: in Section 2 we collect some preliminaries; in Section 3 we study the limit as $p \rightarrow \infty$ in our $p$-Laplacian approximation and prove Theorem 1.1 and Theorem 1.2. At the end of Section 3 we collect examples that illustrate our results. 


\section{Preliminaries.}

2.1. Quantum Graph. We remind here some basic knowledge about quantum graphs, see for instance [3] and references therein.

A graph $\Gamma$ consists of a finite or countable infinite set of vertices $\mathrm{V}(\Gamma)=\left\{\mathrm{v}_{i}\right\}$ and a set of edges $\mathrm{E}(\Gamma)=\left\{\mathrm{e}_{j}\right\}$ connecting the vertices. A graph $\Gamma$ is said a finite graph if the number of edges and the number of vertices are finite. An edge and a vertex on that edge are called incident. We will denote $\mathrm{v} \in \mathrm{e}$ when the edge e and the vertex $\mathrm{v}$ are incident. We define $\mathrm{E}_{\mathrm{v}}(\Gamma)$ as the set of all edges incident to $\mathrm{v}$.

We will assume absence of loops, since if these are present, one can break them into pieces by introducing new intermediate vertices. We also assume absence of multiple edges.

A walk is a sequence of edges $\left\{\mathrm{e}_{1}, \mathrm{e}_{2}, \mathrm{e}_{3}, \ldots\right\}$ in which, for each $i$ (except the last), the end of $\mathrm{e}_{i}$ is the beginning of $\mathrm{e}_{i+1}$. A trail is a walk in which no edge is repeated. A path is a trail in which no vertex is repeated.

From now on we will deal with a connected, compact and metric graph $\Gamma$ :

- A graph $\Gamma$ is a metric graph if

(1) each edge $\mathrm{e}$ is assigned a positive length $\ell_{\mathrm{e}} \in(0,+\infty]$;

(2) for each edge e, a coordinate is assigned to each point of it included their vertices. For that purpose each edge e is identified with an ordered pair $\left(i_{e}, f_{e}\right)$ of vertices, being $i_{e}$ and $f_{e}$ the initial and terminal vertex of e respectively, which no has a meaning of sense when travelling along the path but allows to define coordinates by means of an increasing function

$$
\begin{aligned}
c_{\mathrm{e}}: & \mathrm{e} \\
x & \rightsquigarrow\left[0, \ell_{\mathrm{e}}\right] \\
x & x_{\mathrm{e}}
\end{aligned}
$$

such that, letting $c_{\mathrm{e}}\left(\mathrm{i}_{\mathrm{e}}\right):=0$ and $c_{\mathrm{e}}\left(\mathrm{f}_{\mathrm{e}}\right):=\ell_{\mathrm{e}}$, is exhaustive; $x_{\mathrm{e}}$ is called the coordinate of the point $x \in \mathrm{e}$.

- A graph is said to be connected if a path exists between every pair of vertices, that is, a graph which is connected in the usual topological sense.

- A compact metric graph is a finite metric graph whose edges have all finite length.

If a sequence of edges $\left\{\mathrm{e}_{j}\right\}_{j=1}^{n}$ forms a path, its length is defined as $\sum_{j=1}^{n} \ell_{\mathrm{e}_{j}}$. The length of a metric graph, denoted $\ell(\Gamma)$, is the sum of the length of all its edges.

For two vertices $\mathrm{v}$ and $\hat{\mathrm{v}}$, the distance between $\mathrm{v}$ and $\hat{\mathrm{v}}, d_{\Gamma}(\mathrm{v}, \hat{\mathrm{v}})$, is defined as the minimal length of the paths connecting them. Let us be more precise and consider $x, y$ two points in the graph $\Gamma$.

-if $x, y \in \mathrm{e}$ (they belong to the same edge, note that they can be vertices), we define the distance-in-the-path-e between $x$ and $y$ as

$$
\operatorname{dist}_{\mathrm{e}}(x, y):=\left|y_{\mathrm{e}}-x_{\mathrm{e}}\right|
$$

-if $x \in \mathrm{e}_{a}, y \in \mathrm{e}_{b}$, with $\mathrm{e}_{a}$ and $\mathrm{e}_{b}$ different edges, let $P=\left\{\mathrm{e}_{a}, \mathrm{e}_{1}, \ldots, \mathrm{e}_{n}, \mathrm{e}_{b}\right\}$ be a path $(n \geq 0)$ connecting them. Let us call $\mathrm{e}_{0}=\mathrm{e}_{a}$ and $\mathrm{e}_{n+1}=\mathrm{e}_{b}$. Following the definition given above for a path, set $\mathrm{v}_{0}$ the vertex that is the end of $\mathrm{e}_{0}$ and the beginning of $e_{1}$ (note that these vertices need not be the terminal and the initial vertices of the edges that are taken into account), and $\mathrm{v}_{n}$ the vertex that is the end of $\mathrm{e}_{n}$ and the beginning of $\mathrm{e}_{n+1}$. We will say that the distance-in-the-path-P 
between $x$ and $y$ is equal to

$$
\operatorname{dist}_{\mathrm{e}_{0}}\left(x, \mathrm{v}_{0}\right)+\sum_{1 \leq j \leq n} \ell_{\mathrm{e}_{j}}+\operatorname{dist}_{\mathrm{e}_{n+1}}\left(\mathrm{v}_{n}, y\right) .
$$

We define the distance between $x$ and $y$, that we will denote by

$$
d_{\Gamma}(x, y),
$$

as the infimum of all the distances-in-paths between $x$ and $y$.

Remark that the distance between two points $x$ and $y$ belonging to the same edge e can be strictly smaller than $\left|y_{\mathrm{e}}-x_{\mathrm{e}}\right|$. This happens when there is a path connecting them (using more edges than e) with length smaller than $\left|y_{\mathrm{e}}-x_{\mathrm{e}}\right|$.

A compact metric graph $\Gamma$ becomes a compact metric measure space with respect to the distance $d_{\Gamma}$.

A function $u$ on a metric graph $\Gamma$ is a collection of functions $[u]_{\mathrm{e}}$ defined on $\left(0, \ell_{\mathrm{e}}\right)$ for all $\mathrm{e} \in \mathrm{E}(\Gamma)$, not just at the vertices as in discrete models.

Throughout this work, $\int_{\Gamma} u(x) d x$ or $\int_{\Gamma} u$ denotes $\sum_{\mathrm{e} \in \mathrm{E}(\Gamma)} \int_{0}^{\ell_{\mathrm{e}}}[u]_{\mathrm{e}}\left(x_{\mathrm{e}}\right) d x_{\mathrm{e}}$.

Let $1 \leq p \leq+\infty$. We say that $u$ belongs to $L^{p}(\Gamma)$ if $[u]_{\mathrm{e}}$ belongs to $L^{p}\left(0, \ell_{\mathrm{e}}\right)$ for all $\mathrm{e} \in \mathrm{E}(\Gamma)$ and

$$
\|u\|_{L^{p}(\Gamma)}^{p}:=\sum_{\mathrm{e} \in \mathrm{E}(\Gamma)}\left\|[u]_{\mathrm{e}}\right\|_{L^{p}\left(0, \ell_{\mathrm{e}}\right)}^{p}<+\infty .
$$

The Sobolev space $W^{1, p}(\Gamma)$ is defined as the space of continuous functions $u$ on $\Gamma$ such that $[u]_{\mathrm{e}} \in W^{1, p}\left(0, \ell_{\mathrm{e}}\right)$ for all $\mathrm{e} \in \mathrm{E}(\Gamma)$ and

$$
\|u\|_{W^{1, p}(\Gamma)}^{p}:=\sum_{\mathrm{e} \in \mathrm{E}(\Gamma)}\left\|[u]_{\mathrm{e}}\right\|_{L^{p}\left(0, \ell_{\mathrm{e}}\right)}^{p}+\left\|[u]_{\mathrm{e}}^{\prime}\right\|_{L^{p}\left(0, \ell_{\mathrm{e}}\right)}^{p}<+\infty .
$$

The space $W^{1, p}(\Gamma)$ is a Banach space for $1 \leq p \leq \infty$. It is reflexive for $1<p<\infty$ and separable for $1 \leq p<\infty$. Observe that the continuity condition in the definition of $W^{1, p}(\Gamma)$ means that for each $\mathrm{v} \in \mathrm{V}(\Gamma)$, the function on all edges e $\in \mathrm{E}_{\mathrm{v}}(\Gamma)$ assume the same value at $\mathrm{v}$.

Let $\Gamma$ be a compact graph and $1<p<+\infty$. The injection $W^{1, p}(\Gamma) \subset C(\bar{\Gamma})$ is compact.

A quantum graph is a metric graph $\Gamma$ equipped with a differential operator acting on the edges accompanied by vertex conditions. In this work, we will consider the $p$-Laplacian differential operators given by

$$
-\Delta_{p} u(x):=-\left(\left|u^{\prime}(x)\right|^{p-2} u^{\prime}(x)\right)^{\prime}, \quad \text { with } p>1,
$$

on each edge.

The Monge-Kantorovich problem. Fix $\mu, \nu \in \mathcal{M}^{+}(\Gamma)$ satisfying the mass balance condition

$$
\mu(\Gamma)=\nu(\Gamma)
$$

The Monge-Kantorovich problem is the minimization problem

$$
\min \left\{\int_{\Gamma \times \Gamma} d_{\Gamma}(x, y) d \gamma(x, y): \gamma \in \Pi(\mu, \nu)\right\},
$$

where $\Pi(\mu, \nu):=\left\{\right.$ Radon measures $\gamma$ in $\left.\Gamma \times \Gamma: \pi_{1} \# \gamma=\mu, \pi_{2} \# \gamma=\nu\right\}$. The elements $\gamma \in \Pi(\mu, \nu)$ are called transport plans between $\mu$ and $\nu$, and a minimizer 
$\gamma^{*}$ an optimal transport plan. Since $d$ is lower semicontinuous, there are optimal plans, that is,

$$
\exists \gamma^{*}=\arg \min _{\gamma \in \Pi(\mu, \nu)} \int_{\Gamma \times \Gamma} d_{\Gamma}(x, y) d \gamma(x, y) .
$$

The Monge-Kantorovich problem has a dual formulation that can be stated in this case as follows (see for instance [16, Theorem 1.14]).

Kantorovich-Rubinstein's Theorem. Let $\mu, \nu \in \mathcal{M}^{+}(\Gamma)$ be two measures satisfying the mass balance condition (2.1). Then,

$\min \left\{\int_{\Gamma \times \Gamma} d_{\Gamma}(x, y) d \gamma(x, y): \gamma \in \Pi(\mu, \nu)\right\}=\sup \left\{\int_{\Gamma} u d(\mu-\nu): u \in K_{d_{\Gamma}}(\Gamma)\right\}$,

where $K_{d_{\Gamma}}(\Gamma):=\left\{u: \Gamma \mapsto \mathbb{R}: u(y)-u(x) \leq d_{\Gamma}(y, x)\right\}$. Moreover, there exists $u \in$ $K_{d_{\Gamma}}(\Gamma)$ such that

$$
\int_{\Gamma} u d(\mu-\nu)=\sup \left\{\int_{\Gamma} v d(\mu-\nu): v \in K_{d_{\Gamma}}(\Gamma)\right\} .
$$

Such maximizers are called Kantorovich potentials.

Example. As an example of metric graph in connection with this mass transport problem we can consider the road network in a country which can be consider as a metric graph $\Gamma$ in which $V(\Gamma)$ corresponds to the set of important cities and $E(\Gamma)$ to the roads connecting these cities. Then, for $x \in \mathrm{e} \in E(\Gamma), x_{\mathrm{e}} \in\left(0, \ell_{\mathrm{e}}\right)$, that represents the cost of transporting a unit mass from the city $\mathrm{i}_{\mathrm{e}}$ to $x$ on the road e, could be given by the distance on that road, but we can also take into account the possible tolls by changing the parametrization of the edges, expanding them according to the size of the toll in that road.

\section{The $p$-LAPLACiAn APPROXimation.}

Theorem 3.1. Let $\mu, \nu \in \mathcal{M}^{+}(\Gamma)$ be two measures satisfying the mass balance condition (2.1). Take $\eta=\mu-\nu$. Consider the functional $\mathcal{F}_{p}: W^{1, p}(\Gamma) \rightarrow \mathbb{R}$ defined by

$$
\mathcal{F}_{p}(u):=\frac{1}{p} \int_{\Gamma}\left|u^{\prime}\right|^{p}-\int_{\Gamma} u d \eta .
$$

Then, there exists a minimizer $u_{p}$ of the functional $\mathcal{F}_{p}$ in the set

$$
S_{p}:=\left\{u \in W^{1, p}(\Gamma): \int_{\Gamma} u=0\right\} .
$$

Moreover, a minimizer $u_{p}$ is a weak solution of the problem

$$
\left\{\begin{array}{l}
-\left(\left|u^{\prime}\right|^{p-2} u^{\prime}\right)^{\prime}=\eta \quad \text { on edges } \\
\sum_{\mathrm{e} \in \mathrm{E}_{\mathrm{v}}(\Gamma)}\left|\frac{\partial u}{\partial x_{\mathrm{e}}}\right|^{p-2} \frac{\partial u}{\partial x_{\mathrm{e}}}(\mathrm{v})=0 \quad \text { on vertices },
\end{array}\right.
$$

in the sense that $u_{p} \in W^{1, p}(\Gamma)$ and

$$
\int_{\Gamma}\left|u_{p}^{\prime}\right|^{p-2} u_{p}^{\prime} \varphi^{\prime}=\int_{\Gamma} \varphi d \eta
$$

for every $\varphi \in W^{1, p}(\Gamma)$. 
Observe that in the boundary condition in (3.1) the derivatives are taken in the direction away from the vertex.

Proof. Let $\left\{u_{n}\right\}$ be a minimizing sequence in $S_{p}$. Since $\int_{\Omega} u_{n}=0$, there exists $x_{n} \in \Gamma$ such that $u_{n}\left(x_{n}\right)=0$. Suppose $x_{n} \in \mathrm{e} \in E(\Gamma)$. Then, for $x \in \mathrm{e}$,

$$
\begin{aligned}
\left|u_{n}(x)\right|= & \left|\left[u_{n}\right]_{\mathrm{e}}\left(x_{\mathrm{e}}\right)-\left[u_{n}\right]\left(\left(x_{n}\right)_{\mathrm{e}}\right)\right|=\left|\int_{\left(x_{n}\right)_{\mathrm{e}}}^{x_{\mathrm{e}}}\left[u_{n}\right]_{\mathrm{e}}^{\prime}\right| \\
& \leq\left|x_{\mathrm{e}}-\left(x_{n}\right)_{\mathrm{e}}\right|^{1 / p^{\prime}}\left\|\left[u_{n}\right]_{\mathrm{e}}^{\prime}\right\|_{L^{p}\left(0, \ell_{\mathrm{e}}\right)} \leq C\left\|u_{n}^{\prime}\right\|_{L^{p}(\Gamma)},
\end{aligned}
$$

being $C$ independent of $p$. Now, since $u_{n}$ is continuous, we can apply the above argument in the edges that share a vertex with e. Since $\Gamma$ is connected and compact, doing this at all edges, we get

$$
\sup _{x \in \Gamma}\left|u_{n}(x)\right| \leq C\left\|u_{n}^{\prime}\right\|_{L^{p}(\Gamma)} .
$$

Then, as we have

$$
\left|\int_{\Gamma} u d \eta\right| \leq C\|u\|_{L^{\infty}(\Gamma)} \leq C\|u\|_{W^{1, p}(\Gamma)}
$$

we get that $\left\{u_{n}\right\}$ is bounded in $W^{1, p}(\Gamma)$ and hence (using that $\int_{\Gamma} u_{n}=0$ ) we can extract a subsequence $u_{n_{j}} \rightarrow u_{p}$ weakly in $W^{1, p}(\Gamma)$ and uniformly in $\Gamma$. Then, we have $\int_{\Gamma} u_{p}=0$ and

$$
\mathcal{F}_{p}\left(u_{p}\right) \leq \liminf _{j} \mathcal{F}_{p}\left(u_{n_{j}}\right),
$$

and we conclude that $u_{p}$ is the desired minimizer.

To prove that a minimizer is a weak solution to (3.1) we just observe that when we differentiate with respect to $t$ at $t=0$ the function $\mathcal{F}_{p}\left(u_{p}+t \varphi\right)$ we obtain $u_{p} \in W^{1, p}(\Gamma)$ and

$$
\int_{\Gamma}\left|u_{p}^{\prime}\right|^{p-2} u_{p}^{\prime} \varphi^{\prime}=\int_{\Gamma} \varphi d \eta, \quad \forall \varphi \in W^{1, p}(\Gamma)
$$

This ends the proof.

Now, we show that there is a limit as $p \rightarrow \infty$ of the minimizers $u_{p}$.

Lemma 3.2. Let $u_{p}$ be a minimizer of the functional $\mathcal{F}_{p}$ on $S_{p}, p>1$. There exists a subsequence $p_{j} \rightarrow \infty$ such that

$$
u_{p_{j}} \rightrightarrows u_{\infty}
$$

uniformly in $\bar{\Gamma}$. Moreover, the limit $u_{\infty}$ is Lipschitz continuous and $\left\|u_{\infty}^{\prime}\right\|_{L^{\infty}(\Gamma)} \leq 1$.

Proof. Along this proof we will denote by $C$ a constant independent of $p$ that may change from one line to another.

Our first aim is to prove that the $L^{p}$-norm of the gradient of $u_{p}$ is bounded independently of $p$. Since $u_{p}$ is a minimizer of $\mathcal{F}_{p}$ on $S_{p}$, then,

$$
\mathcal{F}_{p}\left(u_{p}\right)=\int_{\Gamma} \frac{\left|u_{p}^{\prime}\right|^{p}}{p}-\int_{\Gamma} u_{p} d \eta \leq \mathcal{F}_{p}(0)=0 .
$$

That is,

$$
\int_{\Gamma} \frac{\left|u_{p}^{\prime}\right|^{p}}{p} \leq \int_{\Gamma} u_{p} d \eta
$$


Now, from the same arguments leading to (3.2), we obtain

$$
\int_{\Gamma} u_{p} d \eta \leq C\left\|u_{p}^{\prime}\right\|_{L^{p}(\Gamma)}
$$

Then we get

$$
\int_{\Gamma} \frac{\left|u_{p}^{\prime}\right|^{p}}{p} \leq C\left\|u_{p}^{\prime}\right\|_{L^{p}(\Gamma)}
$$

From this inequality, and using that $(p C)^{1 /(p-1)} \rightarrow 1$ (since $C$ is independent of $p$ ) we obtain that

$$
\left\|u_{p}^{\prime}\right\|_{L^{p}(\Gamma)} \leq C
$$

with $C$ a constant independent of $p$. Now, by (3.2) and (3.3), we obtain that

$$
\sup _{x \in \Gamma}\left|u_{p}(x)\right| \leq C,
$$

with $C$ a constant independent of $p$.

Using this uniform bound, we prove uniform convergence of a sequence $u_{p_{j}}$. In fact, we take $m$ such that $1<m \leq p$ and obtain the following bound

$$
\begin{aligned}
\left\|u_{p}^{\prime}\right\|_{L^{m}(\Gamma)} & =\left(\int_{\Gamma}\left|u_{p}^{\prime}\right|^{m} \cdot 1\right)^{\frac{1}{m}} \\
& \leq\left[\left(\int_{\Gamma}\left|u_{p}^{\prime}\right|^{p}\right)^{\frac{m}{p}}\left(\int_{\Gamma} 1\right)^{\frac{p-m}{p}}\right]^{\frac{1}{m}} \\
& \leq C \ell(\Gamma)^{\frac{p-m}{p m}} \leq C,
\end{aligned}
$$

the constants $C$ being independent of $p$. We have proved that $\left\{u_{p}\right\}_{p>1}$ is bounded in $W^{1, m}(\Gamma)$, so we can obtain a weakly convergent sequence $u_{p_{j}} \rightarrow u_{\infty} \in W^{1, m}(\Gamma)$ with $p_{j} \rightarrow+\infty$. Since $W^{1, p}(\Gamma)$ is compactly embedded in $C(\bar{\Gamma})$ and $u_{p_{j}} \rightarrow u_{\infty} \in$ $W^{1, p}(\Gamma)$, we obtain $u_{p_{j}} \rightarrow u_{\infty}$ uniformly in $\bar{\Gamma}$. Using a diagonal procedure we conclude the existence of sequence $u_{p_{j}}$ that is weakly convergent in $W^{1, m}(\Gamma)$ for every $m$.

Finally, let us show that the limit function $u_{\infty}$ is Lipschitz. In fact, we have that

$$
\left(\int_{\Gamma}\left|u_{\infty}^{\prime}\right|^{m}\right)^{\frac{1}{m}} \leq \liminf _{p_{j} \rightarrow+\infty}\left(\int_{\Gamma}\left|u_{p_{j}}^{\prime}\right|^{m}\right)^{\frac{1}{m}} \leq \ell(\Gamma)^{\frac{1}{m}} .
$$

Now, we take $m \rightarrow \infty$ to obtain $\left\|u_{\infty}^{\prime}\right\|_{L^{\infty}(\Gamma)} \leq 1$. So, we have proved that $u_{\infty} \in$ $W^{1, \infty}(\Gamma)$, that is, $u_{\infty}$ is a Lipschitz function and $\left\|u_{\infty}^{\prime}\right\|_{L^{\infty}(\Gamma)} \leq 1$.

Theorem 3.3. Any uniform limit $u_{\infty}$ of a sequence $u_{p_{j}}$ is a Kantorovich potential for the optimal transport problem of $\mu$ to $\nu$ with the cost given $d_{\Gamma}$, that is, it holds that

$$
\begin{aligned}
\min & \left\{\int_{\Gamma \times \Gamma} d_{\Gamma}(x, y) d \gamma(x, y): \gamma \in \Pi(\mu, \nu)\right\} \\
& =\sup \left\{\int_{\Gamma} u d(\nu-\mu): u \in K_{d_{\Gamma}}(\Gamma)\right\}=\int_{\Gamma} u_{\infty} d(\mu-\nu) .
\end{aligned}
$$


Proof. By the Kantorovich-Rubinstein's Theorem, we only need to show the last equality. To do that, first let us see that

$$
K_{d_{\Gamma}}(\Gamma)=\left\{u \in W^{1, \infty}(\Gamma):\left\|u^{\prime}\right\|_{L^{\infty}(\Gamma)} \leq 1\right\} .
$$

It is easy to see that

$$
K_{d_{\Gamma}}(\Gamma) \subset \operatorname{Lip}_{d_{\Gamma}}(\Gamma)=\left\{u \in W^{1, \infty}(\Gamma):\left\|u^{\prime}\right\|_{L^{\infty}(\Gamma)} \leq 1\right\} .
$$

Let us see the reverse inclusion. Let

$$
u \in\left\{u \in W^{1, \infty}(\Gamma):\left\|u^{\prime}\right\|_{L^{\infty}(\Gamma)} \leq 1\right\}
$$

and $x, y \in \Gamma$ with $x \in \mathrm{e}_{a}$ and $y \in \mathrm{e}_{b}$. Suppose that $d_{\Gamma}(x, y)$ is attained at the path $\left\{\mathrm{e}_{0}, \mathrm{e}_{1}, \ldots, \mathrm{e}_{n}, \mathrm{e}_{n+1}\right\}, n \geq-1$, where $\mathrm{e}_{a}=\mathrm{e}_{0}$ and $\mathrm{e}_{b}=\mathrm{e}_{n+1}$. Suppose $\mathrm{f}_{\mathrm{e}_{0}}$ is the vertex that is the end of $e_{0}$ and the beginning of $e_{1}$, and $i_{e_{n+1}}$ the vertex that is the end of $\mathrm{e}_{n}$ and the beginning of $\mathrm{e}_{n+1}$, for other cases the argument is similar. Then, we have

$$
\begin{array}{ll}
d_{\Gamma}(x, y)=\left|y_{\mathrm{e}_{0}}-x_{\mathrm{e}_{0}}\right| & \text { if } n=-1, \\
d_{\Gamma}(x, y)=l_{\mathrm{e}_{0}}-x_{\mathrm{e}_{0}}+\sum_{1 \leq i \leq n} \ell_{\mathrm{e}_{i}}+y_{\mathrm{e}_{n+1}} & \text { if } n \geq-1 .
\end{array}
$$

Now, if $n=-1$

$$
|u(x)-u(y)|=\left|[u]_{\mathrm{e}_{0}}\left(x_{\mathrm{e}_{0}}\right)-[u]_{\mathrm{e}_{0}}\left(y_{\mathrm{e}_{0}}\right)\right| \leq\left|y_{\mathrm{e}_{0}}-x_{\mathrm{e}_{0}}\right|=d_{\Gamma}(x, y),
$$

and if $n \geq 0$,

$$
\begin{gathered}
\left|u(x)-u\left(\mathrm{f}_{\mathrm{e}_{0}}\right)\right|=\left|[u]_{\mathrm{e}_{0}}\left(x_{\mathrm{e}_{0}}\right)-[u]_{\mathrm{e}_{0}}\left(\ell_{\mathrm{e}_{0}}\right)\right| \leq \ell_{\mathrm{e}_{0}}-x_{\mathrm{e}_{0}}, \\
\left|u\left(\mathrm{i}_{\mathrm{e}_{i}}\right)-u\left(\mathrm{f}_{\mathrm{e}_{i}}\right)\right|=\left|[u]_{\mathrm{e}_{i}}(0)-[u]_{\mathrm{e}_{i}}\left(\ell_{\mathrm{e}_{i}}\right)\right| \leq \ell_{\mathrm{e}_{i+1}}, \quad 1 \leq i \leq n,
\end{gathered}
$$

and

Hence,

$$
\left|u\left(\mathrm{i}_{\mathrm{e}_{n+1}}\right)-u(y)\right|=\left|[u]_{\mathrm{e}_{n+1}}(0)-[u]_{\mathrm{e}_{n+1}}\left(y_{\mathrm{e}_{n+1}}\right)\right| \leq y_{\mathrm{e}_{n+1}} .
$$

$$
\begin{gathered}
|u(x)-u(y)| \leq\left|u(x)-u\left(\mathrm{f}_{\mathrm{e}_{0}}\right)\right|+\sum_{1 \leq i \leq n}\left|u\left(\mathrm{i}_{\mathrm{e}_{i}}\right)-u\left(\mathrm{f}_{\mathrm{e}_{i}}\right)\right|+\left|u\left(\mathrm{i}_{\mathrm{e}_{n+1}}\right)-u(y)\right| \\
\leq \ell_{\mathrm{e}_{0}}-x_{\mathrm{e}_{0}}+\sum_{1 \leq i \leq n} \ell_{\mathrm{e}_{i}}+y_{\mathrm{e}_{n+1}}=d_{\Gamma}(x, y) .
\end{gathered}
$$

Consequently, (3.5) holds.

Due to (3.5), we just need to show that

$$
\sup \left\{\int_{\Gamma} v d(\mu-\nu): v \in \operatorname{Lip}_{d_{\Gamma}}\right\}=\int_{\Gamma} u_{\infty} d(\mu-\nu) .
$$

Given $v \in \operatorname{Lip}_{d_{\Gamma}}(\Gamma)$, if we define

$$
\tilde{v}:=v-\frac{1}{\ell(\Gamma)} \int v d x
$$

We have $\tilde{v} \in S_{p}$, then

$$
\begin{aligned}
\mathcal{F}_{p}\left(u_{p}\right)= & \int_{\Gamma} \frac{\left|u_{p}^{\prime}\right|^{p}}{p}-\int_{\Gamma} u_{p} d \eta \leq \mathcal{F}_{p}(\tilde{v}) \\
& =\int_{\Gamma} \frac{\left|v^{\prime}\right|^{p}}{p}-\int_{\Gamma} v d \eta \leq \frac{1}{p} \ell(\Gamma)-\int_{\Omega} v d \eta .
\end{aligned}
$$


Therefore,

$$
-\int_{\Gamma} u_{p} d \eta \leq \int_{\Gamma} \frac{\left|u_{p}^{\prime}\right|^{p}}{p}-\int_{\Gamma} u_{p} d \eta \leq \int_{\Gamma} \frac{\left|v^{\prime}\right|^{p}}{p}-\int_{\Gamma} v d \eta \leq \frac{1}{p} \ell(\Gamma)-\int_{\Gamma} v d \eta .
$$

Taking limits as $p \rightarrow \infty$ we obtain

$$
\int_{\Gamma} u_{\infty} d(\mu-\nu) \geq \int_{\Gamma} v d(\mu-\nu)
$$

and consequently, we get

$$
\int_{\Gamma} u_{\infty} d(\mu-\nu) \geq \sup \left\{\int_{\Gamma} v d(\mu-\nu): v \in \operatorname{Lip}_{d_{\Gamma}}(\Gamma)\right\},
$$

from where it follows $(3.6)$, since $u_{\infty} \in \operatorname{Lip}_{d}(\Gamma)$.

In order to find the transport density we need the following result.

Lemma 3.4. Let $\mu=\mu^{+}-\mu^{-}, \mu^{ \pm}$positive Radon measure in $(a, b)$, and $\alpha, \beta \in \mathbb{R}$ satisfying

$$
\int_{a}^{b} d \mu+\alpha+\beta=0
$$

(i) For any $p>1$ let $v_{p}$ be a weak solution of the problem

$$
\left\{\begin{array}{l}
-\left(\left|v^{\prime}\right|^{p-2} v^{\prime}\right)^{\prime}=\mu \quad \text { in }(a, b) \\
\left(\left|v^{\prime}\right|^{p-2} v^{\prime}\right)(a)=-\alpha \\
\left(\left|v^{\prime}\right|^{p-2} v^{\prime}\right)(b)=\beta
\end{array}\right.
$$

If

$$
v_{p} \rightrightarrows v_{\infty}
$$

uniformly in $[a, b]$ with $\left\|v_{\infty}^{\prime}\right\|_{L^{\infty}(a, b)} \leq 1$, then $v_{\infty}$ is a Kantorovich potential for the optimal transport problem of $\eta^{+}:=\mu^{+}+\left(\alpha^{+} \delta_{a}+\beta^{+} \delta_{b}\right)$ to $\eta^{-}:=$ $\mu^{-}+\left(\alpha^{-} \delta_{a}+\beta^{-} \delta_{b}\right)$ with the cost given by the Euclidean distance.

(ii) If there exists a nonnegative function $\mathbf{a} \in L^{\infty}(a, b)$ and a Lipschitz continuous function $u$, with $\left\|u^{\prime}\right\|_{L^{\infty}(a, b)} \leq 1$, such that $u$ is a weak solution of

$$
\left\{\begin{array}{l}
-\left(\mathbf{a} u^{\prime}\right)^{\prime}=\mu \quad \text { in }(a, b), \\
\mathbf{a} u^{\prime}(a)=-\alpha \\
\mathbf{a} u^{\prime}(b)=\beta
\end{array}\right.
$$

in the sense

$$
\int_{a}^{b} \mathbf{a} u^{\prime} \varphi^{\prime}-\int_{a}^{b} \varphi d \mu=\alpha \varphi(a)+\beta \varphi(a)
$$

for all $\varphi \in W^{1, \infty}\left(0, \ell_{\mathrm{e}}\right)$, and verifies

$$
\left|u^{\prime}\right|=1 \quad \text { on }\{\mathbf{a}>0\},
$$

then $u$ is a Kantorovich potential for the optimal transport problem of $\eta^{+}$ to $\eta^{-}$with the cost given by the Euclidean distance. 
Proof. (i) Since $v_{p}$ be a weak solution of the problem (3.7), we have

$$
\int_{a}^{b}\left(\left|v_{p}^{\prime}\right|^{p-2} v_{p}^{\prime}\right) w^{\prime}=\int_{a}^{b} w d \mu+\alpha w(a)+\beta w(b), \quad \forall w \in W^{1, p}(] a, b[) .
$$

Taking $w=v_{p}$ in (3.9), we obtain

$$
\int_{a}^{b}\left|v_{p}^{\prime}\right|^{p}=\int_{a}^{b} v_{p} d \mu+\alpha v_{p}(a)+\beta v_{p}(b) \leq C .
$$

Given $v \in W^{1, \infty}(] a, b[)$ with $\left\|v^{\prime}\right\|_{\infty} \leq 1$, taking $w=v_{p}-v$ in (3.9), we get

$$
\begin{aligned}
& \int_{a}^{b}\left|v_{p}^{\prime}\right|^{p}-\int_{a}^{b}\left(\left|v_{p}^{\prime}\right|^{p-2} v_{p}^{\prime}\right) v^{\prime} \\
& \quad=\int_{a}^{b}\left(v_{p}-v\right) d \mu+\alpha\left(v_{p}(a)-v(a)\right)+\beta\left(v_{p}(b)-v(b)\right) .
\end{aligned}
$$

Hence

$$
\int_{a}^{b} v_{p} \eta-\int_{a}^{b} v \eta=\int_{a}^{b}\left|v_{p}^{\prime}\right|^{p}-\int_{a}^{b}\left(\left|v_{p}^{\prime}\right|^{p-2} v_{p}^{\prime}\right) v^{\prime}
$$

Now, by Young's inequality, we have

$$
\begin{aligned}
& \int_{a}^{b}\left(\left|v_{p}^{\prime}\right|^{p-2} v_{p}^{\prime}\right) v^{\prime} \leq \frac{p-1}{p} \int_{a}^{b}\left|v_{p}^{\prime}\right|^{p}+\frac{1}{p} \int_{a}^{b}\left|v^{\prime}\right|^{p} \\
& \quad \leq \frac{p-1}{p} \int_{a}^{b}\left|v_{p}^{\prime}\right|^{p}+\frac{1}{p}(b-a) .
\end{aligned}
$$

Therefore, we obtain

$$
\int_{a}^{b} v_{p} d \eta-\int_{a}^{b} v d \eta \geq \frac{1}{p} \int_{a}^{b}\left|v_{p}^{\prime}\right|^{p}-\frac{1}{p}(b-a) \geq-\frac{1}{p}(b-a) .
$$

Taking limits as $p \rightarrow \infty$ we get

$$
\int_{a}^{b} v_{\infty} d \eta \geq \int_{a}^{b} v d \eta
$$

and consequently

$$
\int_{a}^{b} v_{\infty} d \eta=\sup \left\{\int_{a}^{b} v d \eta: v \in W^{1, \infty}(] a, b[) \text { with }\left\|v^{\prime}\right\|_{\infty} \leq 1\right\} .
$$

This ends the proof of (i).

(ii) Taking $u$ as test function in (3.8), we get that

$$
\int_{a}^{b} u d \mu+\alpha u(0)+\beta u(b)=\int_{a}^{b} \mathbf{a} .
$$

Take now a Lipschitz continuous function $\hat{u}$, with $\left\|\hat{u}^{\prime}\right\|_{L^{\infty}(a, b)} \leq 1$, as test function in (3.8). Then

$$
\begin{aligned}
& \int_{a}^{b} \hat{u} d \mu+\alpha \hat{u}(a)+\beta \hat{u}(b)=\int_{a}^{b} \mathbf{a} u^{\prime} \hat{u}^{\prime} \\
& \leq \int_{a}^{b} \mathbf{a}=\int_{a}^{b} u d \mu+\alpha u(0)+\beta u(b),
\end{aligned}
$$

which gives the assertion. 
Suppose now that $\eta$ is as in (1.1) and take $u_{p}$ as in Theorem 3.1. Then, $u_{p} \in$ $W^{1, p}(\Gamma)$ and

$$
\int_{\Gamma}\left|u_{p}^{\prime}\right|^{p-2} u_{p}^{\prime} \varphi^{\prime}=\sum_{\mathrm{e} \in \mathrm{E}_{\mathrm{v}}(\Gamma)} \int_{0}^{\ell_{\mathrm{e}}}[\varphi]_{\mathrm{e}} d \mu_{\mathrm{e}}+\sum_{\mathrm{v} \in \mathrm{V}(\Gamma)} a_{\mathrm{v}} \varphi(\mathrm{v})
$$

for every $\varphi \in W^{1, p}(\Gamma)$. For a fixed edge e $\in E(\Gamma)$, we define the distribution $\eta_{p, \mathrm{e}}$ in $\mathbb{R}$ as

$$
\left\langle\eta_{p, \mathrm{e}}, \varphi\right\rangle:=\int_{0}^{\ell_{\mathrm{e}}} \mid\left[u_{p}\right]_{\mathrm{e}}^{\left.\right|^{p-2}}\left[u_{p}\right]_{\mathrm{e}}^{\prime} \varphi^{\prime}-\int_{0}^{\ell_{\mathrm{e}}} \varphi d \mu_{\mathrm{e}}, \quad \text { for all } \varphi \in C_{c}^{\infty}(\mathbb{R}) .
$$

Theorem 3.5. For $u_{p}$ as above, measures $\eta_{p, \mathrm{e}}$, and $u_{\infty}$ as in Lemma 3.2, we have:

(1) For each edge $\mathrm{e} \in E(\Gamma)$ the following facts hold:

(a) $\eta_{p, \mathrm{e}}$ is a Radon measure on $\mathbb{R}$ supported on $\left\{0, \ell_{\mathrm{e}}\right\}$, and consequently

$$
\eta_{p, \mathrm{e}}=a_{p, \mathrm{e}, \mathrm{i}_{\mathrm{e}}} \delta_{0}+a_{p, \mathrm{e}, \mathrm{f}_{\mathrm{e}}} \delta_{\ell_{\mathrm{e}}}, \quad a_{p, \mathrm{e}, \mathrm{i}_{\mathrm{e}}}, a_{p, \mathrm{e}, \mathrm{f}_{\mathrm{e}}} \in \mathbb{R} ;
$$

(b) $\left[u_{p}\right]_{\mathrm{e}}$ is a weak solution of

$$
\left\{\begin{array}{l}
-\left(\left|u^{\prime}\right|^{p-2} u^{\prime}\right)^{\prime}=\mu_{\mathrm{e}} \quad \text { in }\left(0, \ell_{\mathrm{e}}\right), \\
\left|\frac{\partial u}{\partial x_{\mathrm{e}}}\right|^{p-2} \frac{\partial u}{\partial x_{\mathrm{e}}}(0)=-a_{p, \mathrm{e}, \mathrm{i}_{\mathrm{e}}}, \\
\left|\frac{\partial u}{\partial x_{\mathrm{e}}}\right|^{p-2} \frac{\partial u}{\partial x_{\mathrm{e}}}\left(\ell_{\mathrm{e}}\right)=a_{p, \mathrm{e}, \mathrm{f}_{\mathrm{e}}} ;
\end{array}\right.
$$

(c) for a subsequence $p_{i} \rightarrow+\infty$,

$$
\left(a_{p_{i}, \mathrm{e}, \mathrm{i}_{\mathrm{e}}}, a_{p_{i}, \mathrm{e}, \mathrm{f}_{\mathrm{e}}}\right) \rightarrow\left(a_{\infty, \mathrm{e}, \mathrm{i}_{\mathrm{e}}}, a_{\infty, \mathrm{e}, \mathrm{f}_{\mathrm{e}}}\right) ;
$$

(d) $\left[u_{\infty}\right]_{\mathrm{e}}$ is a Kantorovich potential for the optimal transport problem of $\mu_{\mathrm{e}}^{+}+\left(a_{\infty, \mathrm{e}, \mathrm{i}_{\mathrm{e}}}\right)^{+} \delta_{0}+\left(a_{\infty, \mathrm{e}, \mathrm{f}_{\mathrm{e}}}\right)^{+} \delta_{\ell_{\mathrm{e}}}$ to $\mu_{\mathrm{e}}^{-}+\left(a_{\infty, \mathrm{e}, \mathrm{i}_{\mathrm{e}}}\right)^{-} \delta_{0}+\left(a_{\infty, \mathrm{e}, \mathrm{f}_{\mathrm{e}}}\right)^{-} \delta_{\ell_{\mathrm{e}}}$ with the cost given by the Euclidean distance.

(2) $\sum_{\mathrm{e} \in \mathrm{E}_{\mathrm{v}}(\Gamma)} a_{\infty, \mathrm{e}, \mathrm{v}}=a_{\mathrm{v}}$ for any $\mathrm{v} \in \mathrm{V}(\Gamma)$.

Proof. (1a) Given $\varphi \in C_{c}^{\infty}(\mathbb{R})$ supported on $\mathbb{R} \backslash\left\{0, \ell_{\mathrm{e}}\right\}$, we have

$$
\int_{0}^{\ell_{\mathrm{e}}}\left|\left[u_{p}\right]_{\mathrm{e}}^{\prime}\right|^{p-2}\left[u_{p}\right]_{\mathrm{e}}^{\prime} \varphi^{\prime}-\int_{0}^{\ell_{\mathrm{e}}} \varphi d \mu_{\mathrm{e}}=0 .
$$

Therefore, $\eta_{p, \text { e }}$ defines a Radon measure on $\mathbb{R}$ supported on $\left\{0, \ell_{\mathrm{e}}\right\}$, and consequently there exist $a_{p, \mathrm{i}_{\mathrm{e}}}, a_{p, \mathrm{f}_{\mathrm{e}}} \in \mathbb{R}$ such that

$$
\eta_{p, \mathrm{e}}=a_{p, \mathrm{e}, \mathrm{i}_{\mathrm{e}}} \delta_{0}+a_{p, \mathrm{e}, \mathrm{f}_{\mathrm{e}}} \delta_{\ell_{\mathrm{e}}} .
$$

(1b) Given $\varphi \in W^{1, p}\left(0, \ell_{\mathrm{e}}\right)$, let $\varphi_{n} \in C_{c}^{\infty}(\mathbb{R})$ such that

$$
\varphi_{n \mid\left(0, \ell_{\mathrm{e}}\right)} \rightarrow \varphi
$$

in $W^{1, p}\left(0, \ell_{\mathrm{e}}\right)$. Then

$$
\int_{0}^{\ell_{\mathrm{e}}} \mid\left[u_{p}\right]_{\mathrm{e}}^{\left.\right|^{p-2}}\left[u_{p}\right]_{\mathrm{e}}^{\prime} \varphi_{n}^{\prime}-\int_{0}^{\ell_{\mathrm{e}}} \varphi_{n} d \mu_{\mathrm{e}}=a_{p, \mathrm{e}, \mathrm{f}_{\mathrm{e}}} \varphi_{n}\left(\ell_{\mathrm{e}}\right)+a_{p, \mathrm{e}, \mathrm{i}_{\mathrm{e}}} \varphi_{n}(0) .
$$


Hence, taking limits as $n \rightarrow \infty$, we obtain that

$$
\int_{0}^{\ell_{\mathrm{e}}}\left|\left[u_{p}\right]_{\mathrm{e}}^{\prime}\right|^{p-2}\left[u_{p}\right]_{\mathrm{e}}^{\prime} \varphi^{\prime}-\int_{0}^{\ell_{\mathrm{e}}} \varphi d \mu_{\mathrm{e}}=a_{p, \mathrm{e}, \mathrm{f}_{\mathrm{e}}} \varphi\left(\ell_{\mathrm{e}}\right)+a_{p, \mathrm{e}, \mathrm{i}_{\mathrm{e}}} \varphi(0) .
$$

Therefore, $\left[u_{p}\right]_{\mathrm{e}}$ is a weak solution of (3.10).

(1c) Let us see that $\left\{a_{p, \mathrm{e}, \mathrm{i}_{\mathrm{e}}}\right\}_{p>1}$ and $\left\{a_{p, \mathrm{e}, \mathrm{f}_{\mathrm{e}}}\right\}_{p>1}$ are bounded. Taking in (3.11) $\varphi=\left[u_{p}\right]_{\mathrm{e}}$, since by $(3.4),\left[u_{p}\right]_{\mathrm{e}}$ is uniformly bounded independent of $p$, we have

$$
\int_{0}^{\ell_{\mathrm{e}}}\left|\left[u_{p}\right]_{\mathrm{e}}^{\prime}\right|^{p} \leq \int_{0}^{\ell_{\mathrm{e}}}\left[u_{p}\right]_{\mathrm{e}} d \mu_{\mathrm{e}}+a_{p, \mathrm{e}, \mathrm{f}_{\mathrm{e}}}\left[u_{p}\right]_{\mathrm{e}}\left(\ell_{\mathrm{e}}\right)+a_{p, \mathrm{e}, \mathrm{i}_{\mathrm{e}}}\left[u_{p}\right]_{\mathrm{e}}(0) \leq C,
$$

for every $p>1$.

On the other hand, taking $\varphi(x)=x$ in (3.11), we get

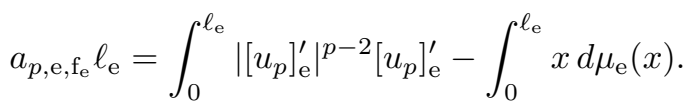

Then, by (3.12) and using Hölder's inequality, we get that $\left\{a_{p, \mathrm{e}, \mathrm{f}_{\mathrm{e}}}\right\}_{p>1}$ is bounded. Finally, taking $\varphi=1$ in (3.11) we get $\left\{a_{p, \mathrm{e}, \mathrm{i}_{\mathrm{e}}}\right\}$ is bounded.

(1d) From the compatibility condition of problem (3.10), we have

$$
\int_{0}^{\ell_{\mathrm{e}}} d \mu_{\mathrm{e}}+a_{p, \mathrm{e}, \mathrm{i}_{\mathrm{e}}}+a_{p, \mathrm{e}, \mathrm{f}_{\mathrm{e}}}=0 .
$$

Taking limit as $p \rightarrow \infty$ we obtain

$$
\int_{0}^{\ell_{\mathrm{e}}} d \mu_{\mathrm{e}}+a_{\infty, \mathrm{e}, \mathrm{i}_{\mathrm{e}}}+a_{\infty, \mathrm{e}, \mathrm{f}_{\mathrm{e}}}=0 .
$$

Then, by Lemma 3.4, we have that $\left[u_{\infty}\right]_{\mathrm{e}}$ is a Kantorovich potential for the optimal transport problem of $\left[\mu^{+}\right]_{\mathrm{e}}+\left(a_{\infty, \mathrm{e}, \mathrm{i}_{\mathrm{e}}}\right)^{+} \delta_{0}+\left(a_{\infty, \mathrm{e}, \mathrm{f}_{\mathrm{e}}}\right)^{+} \delta_{\ell_{\mathrm{e}}}$ to $\left[\mu^{-}\right]_{\mathrm{e}}+\left(a_{\infty, \mathrm{e}, \mathrm{i}_{\mathrm{e}}}\right)^{-} \delta_{0}+$ $\left(a_{\infty, \mathrm{e}, \mathrm{f}_{\mathrm{e}}}\right)^{-} \delta_{\ell_{\mathrm{e}}}$ with the cost given by the Euclidean distance.

(2) Given $\varphi \in W^{1, p}(\Gamma)$, since $u_{p}$ is a weak solution of (3.1), adding (3.11) for all $\mathrm{e} \in \mathrm{E}(\Gamma)$

$$
\sum_{\mathrm{e} \in \mathrm{E}(\Gamma)}\left(a_{p, \mathrm{e}, \mathrm{f}_{\mathrm{e}}}[\varphi]_{\mathrm{e}}\left(\ell_{\mathrm{e}}\right)+a_{p, \mathrm{e}, \mathrm{i}_{\mathrm{e}}}[\varphi]_{\mathrm{e}}(0)\right)=\sum_{\mathrm{v} \in \mathrm{V}(\Gamma)} a_{\mathrm{v}} \varphi(\mathrm{v}) .
$$

Letting $p \rightarrow+\infty$ we get

$$
\sum_{\mathrm{e} \in \mathrm{E}(\Gamma)}\left(a_{\infty, \mathrm{e}, \mathrm{f}_{\mathrm{e}}}[\varphi]_{\mathrm{e}}\left(\ell_{\mathrm{e}}\right)+a_{\infty, \mathrm{e}, \mathrm{i}_{\mathrm{e}}}[\varphi]_{\mathrm{e}}(0)\right)=\sum_{\mathrm{v} \in \mathrm{V}(\Gamma)} a_{\mathrm{v}} \varphi(\mathrm{v}) .
$$

Then, rearranging the terms,

$$
\sum_{\mathrm{v} \in \mathrm{V}(\Gamma)} \sum_{\mathrm{e} \in \mathrm{E}_{\mathrm{v}}(\Gamma)} a_{\infty, \mathrm{e}, \mathrm{v}} \varphi(\mathrm{v})=\sum_{\mathrm{v} \in \mathrm{V}(\Gamma)} a_{\mathrm{v}} \varphi(\mathrm{v}),
$$

from where we get the desired conclusion.

Observe that $a_{\infty, \mathrm{e}, \mathrm{v}}$ are solutions of the following system of linear equations for unknowns $a_{\mathrm{e}, \mathrm{v}}$ :

$$
\left(L_{\mu, a_{\mathrm{v}}}\right)\left\{\begin{array}{l}
\sum_{\mathrm{v} \in \mathrm{e}} a_{\mathrm{e}, \mathrm{v}}=-\int_{0}^{\ell_{\mathrm{e}}} d \mu_{\mathrm{e}} \quad \forall \mathrm{e} \in \mathrm{E}(\Gamma), \\
\sum_{\mathrm{e} \in \mathrm{E}_{\mathrm{v}}(\Gamma)} a_{\mathrm{e}, \mathrm{v}}=a_{\mathrm{v}} \quad \forall \mathrm{v} \in \mathrm{V}(\Gamma) .
\end{array}\right.
$$


For any solution $\left\{a_{\mathrm{e}, \mathrm{v}}\right\}$ of $\left(L_{\mu, a_{\mathrm{v}}}\right)$, we define

$$
\mathcal{C}_{\mu, a_{\mathrm{v}}}\left(\left\{a_{\mathrm{e}, \mathrm{v}}\right\}\right):=\sum_{\mathrm{e} \in \mathrm{E}(\Gamma)} W_{1}\left(\left(\mu_{\mathrm{e}}+\sum_{\mathrm{v} \in \mathrm{e}}\left[a_{\mathrm{e}, \mathrm{v}} \delta_{\mathrm{v}}\right]_{\mathrm{e}}\right)^{+},\left(\mu_{\mathrm{e}}+\sum_{\mathrm{v} \in \mathrm{e}}\left[a_{\mathrm{e}, \mathrm{v}} \delta_{\mathrm{v}}\right]_{\mathrm{e}}\right)^{-}\right),
$$

where

$$
\sum_{\mathrm{v} \in \mathrm{e}}\left[a_{\mathrm{e}, \mathrm{v}} \delta_{\mathrm{v}}\right]_{\mathrm{e}}=a_{\mathrm{e}, \mathrm{i}_{\mathrm{e}}} \delta_{0}+a_{\mathrm{e}, \mathrm{f}_{\mathrm{e}}} \delta_{\ell_{\mathrm{e}}}
$$

and $W_{1}$ is the Wasserstein distance in $\mathbb{R}$ respect to the cost $c(x, y)=|x-y|$. In the next result we shall see how to solve the transport problem in graphs through the $\left\{a_{\infty, \mathrm{e}, \mathrm{v}}\right\}$ and the functional $\mathcal{C}_{\mu, a_{\mathrm{v}}}$.

Theorem 3.6. Let $\eta$ be a measure given by (1.1). Under the assumptions of Theorem 3.5 and for $\left\{a_{\infty, \mathrm{e}, \mathrm{v}}\right\}_{\substack{\mathrm{v} \in \mathrm{v} \in \mathrm{e} \\ \mathrm{E}(\Gamma)}}$ given there, we have that

$$
\left\{a_{\infty, \mathrm{e}, \mathrm{v}}\right\}_{\substack{\mathrm{v} \in \mathrm{v} \in \mathrm{e}(\Gamma) \\ \mathrm{E} \in \mathrm{e}}}^{\arg \min } \in \mathcal{C}_{\mu, a_{\mathrm{v}}}\left(\left\{a_{\mathrm{e}, \mathrm{v}}\right\}\right) .
$$

Moreover

$$
\begin{aligned}
W_{\mu, a_{\mathrm{v}}}= & \min \left\{\int_{\Gamma \times \Gamma} d_{\Gamma}(x, y) d \sigma(x, y): \sigma \in \Pi\left(\eta^{+}, \eta^{-}\right)\right\} \\
& =\int_{\Gamma} u_{\infty}\left(\mu+\sum_{\mathrm{v} \in \mathrm{V}(\Gamma)} a_{\mathrm{v}} \delta_{\mathrm{v}}\right) \\
& =\min _{\left\{a_{\mathrm{e}, \mathrm{v}}\right\} \text { solve }\left(L_{\mu, a_{\mathrm{v}}}\right)} \mathcal{C}_{\mu, a_{\mathrm{v}}}\left(\left\{a_{\mathrm{e}, \mathrm{v}}\right\}\right) \\
& =\mathcal{C}_{\mu, a_{\mathrm{v}}}\left(\left\{a_{\infty, \mathrm{e}, \mathrm{v}}\right\}\right) .
\end{aligned}
$$

Proof. Since $a_{\infty, \mathrm{e}, \mathrm{v}}$ are solutions of $\left(L_{\mu, a_{\mathrm{v}}}\right)$, we have

$$
\int_{\Gamma} u_{\infty}\left(\sum_{\mathrm{v} \in \mathrm{V}(\Gamma)} a_{\mathrm{v}} \delta_{\mathrm{v}}\right)=\sum_{\mathrm{v} \in \mathrm{V}(\Gamma)} a_{\mathrm{v}} u_{\infty}(\mathrm{v})=\sum_{\mathrm{v} \in \mathrm{V}(\Gamma)}\left(\sum_{\mathrm{e} \in \mathrm{E}_{\mathrm{v}}(\Gamma)} a_{\infty, \mathrm{e}, \mathrm{v}}\right) u_{\infty}(\mathrm{v}),
$$

and, rearranging,

$$
\int_{\Gamma} u_{\infty}\left(\sum_{\mathrm{v} \in \mathrm{V}(\Gamma)} a_{\mathrm{v}} \delta_{\mathrm{v}}\right)=\sum_{\mathrm{e} \in \mathrm{E}(\Gamma)} \sum_{\mathrm{v} \in \mathrm{e}} a_{\infty, \mathrm{e}, \mathrm{v}} u_{\infty}(\mathrm{v}) .
$$

Therefore,

$$
\begin{aligned}
\int_{\Gamma} u_{\infty}\left(\mu+\sum_{\mathrm{v} \in \mathrm{V}(\Gamma)} a_{\mathrm{v}} \delta_{\mathrm{v}}\right) \\
=\sum_{\mathrm{e} \in \mathrm{E}(\Gamma)}\left(\int_{0}^{\ell_{\mathrm{e}}}\left[u_{\infty}\right]_{\mathrm{e}} d \mu_{\mathrm{e}}+a_{\infty, \mathrm{e}, \mathrm{e}_{\mathrm{e}}}\left[u_{\infty}\right]_{\mathrm{e}}(0)+a_{\infty, \mathrm{e}, \mathrm{f}_{\mathrm{e}}}\left[u_{\infty}\right]_{\mathrm{e}}\left(\ell_{\mathrm{e}}\right)\right) .
\end{aligned}
$$

Now, since $\left[u_{\infty}\right]_{\mathrm{e}}$ is a Kantorovich potential for the optimal transport problem of $\left[\mu^{+}\right]_{\mathrm{e}}+\left(a_{\infty, \mathrm{e}, \mathrm{i}_{\mathrm{e}}}\right)^{+} \delta_{0}+\left(a_{\infty, \mathrm{e}, \mathrm{f}_{\mathrm{e}}}\right)^{+} \delta_{\ell_{\mathrm{e}}}$ to $\left[\mu^{-}\right]_{\mathrm{e}}+\left(a_{\infty, \mathrm{e}, \mathrm{i}_{\mathrm{e}}}\right)^{-} \delta_{0}+\left(a_{\infty, \mathrm{e}, \mathrm{f}_{\mathrm{e}}}\right)^{-} \delta_{\ell_{\mathrm{e}}}$ with the 
cost given by the Euclidean distance, we have

$$
\begin{aligned}
\int_{0}^{\ell_{\mathrm{e}}} & {\left[u_{\infty}\right]_{\mathrm{e}} d \mu_{\mathrm{e}}+a_{\infty, \mathrm{e}, \mathrm{i}_{\mathrm{e}}}\left[u_{\infty}\right]_{\mathrm{e}}(0)+a_{\infty, \mathrm{e}, \mathrm{f}_{\mathrm{e}}}\left[u_{\infty}\right]_{\mathrm{e}}\left(\ell_{\mathrm{e}}\right) } \\
& =W_{1}\left(\left(\mu_{\mathrm{e}}+\sum_{\mathrm{v} \in \mathrm{e}}\left[a_{\infty, \mathrm{e}, \mathrm{v}} \delta_{\mathrm{v}}\right]_{\mathrm{e}}\right)^{+},\left(\mu_{\mathrm{e}}+\sum_{\mathrm{v} \in \mathrm{e}}\left[a_{\infty, \mathrm{e}, \mathrm{v}} \delta_{\mathrm{v}}\right]_{\mathrm{e}}\right)^{-}\right) .
\end{aligned}
$$

Consequently,

$$
\begin{aligned}
\int_{\Gamma} u_{\infty}\left(\mu+\sum_{\mathrm{v} \in \mathrm{V}(\Gamma)} a_{\mathrm{v}} \delta_{\mathrm{v}}\right) \\
=\sum_{\mathrm{e} \in \mathrm{E}(\Gamma)} W_{1}\left(\left(\mu_{\mathrm{e}}+\sum_{\mathrm{v} \in \mathrm{e}}\left[a_{\infty, \mathrm{e}, \mathrm{v}} \delta_{\mathrm{v}}\right]_{\mathrm{e}}\right)^{+},\left(\mu_{\mathrm{e}}+\sum_{\mathrm{v} \in \mathrm{e}}\left[a_{\infty, \mathrm{e}, \mathrm{v}} \delta_{\mathrm{v}}\right]_{\mathrm{e}}\right)^{-}\right) \\
=\mathcal{C}_{\mu, a_{\mathrm{v}}}\left(\left\{a_{\infty, \mathrm{e}, \mathrm{v}}\right\}\right) .
\end{aligned}
$$

Hence, for

$$
\left\{\tilde{a}_{\mathrm{e}, \mathrm{v}}\right\}_{\mathrm{e} \in \mathrm{v} \in \mathrm{E}(\Gamma)} \in \underset{\left\{a_{\mathrm{e}, \mathrm{v}}\right\} \text { solve }\left(L_{\mu, a_{\mathrm{v}}}\right)}{\arg \min } \mathcal{C}_{\mu, a_{\mathrm{v}}}\left(\left\{a_{\mathrm{e}, \mathrm{v}}\right\}\right)
$$

we have that

$$
\begin{aligned}
\int_{\Gamma} u_{\infty} & \left(\mu+\sum_{\mathrm{v} \in \mathrm{V}(\Gamma)} a_{\mathrm{v}} \delta_{\mathrm{v}}\right) \\
& \geq \sum_{\mathrm{e} \in \mathrm{E}(\Gamma)} W_{1}\left(\left(\mu_{\mathrm{e}}+\sum_{\mathrm{v} \in \mathrm{e}}\left[\tilde{a}_{\mathrm{e}, \mathrm{v}} \delta_{\mathrm{v}}\right]_{\mathrm{e}}\right)^{+},\left(\mu_{\mathrm{e}}+\sum_{\mathrm{v} \in \mathrm{e}}\left[\tilde{a}_{\mathrm{e}, \mathrm{v}} \delta_{\mathrm{v}}\right]_{\mathrm{e}}\right)^{-}\right) \\
& =\mathcal{C}_{\mu, a_{\mathrm{v}}}\left(\left\{\tilde{a}_{\mathrm{e}, \mathrm{v}}\right\}\right) .
\end{aligned}
$$

Given e $\in E(\Gamma)$, let $\gamma_{\mathrm{e}}$ be an optimal transport plan between $\left(\mu\left\llcorner\mathrm{e}+\sum_{\mathrm{v} \in \mathrm{e}} \tilde{a}_{\mathrm{e}, \mathrm{v}} \delta_{\mathrm{v}}\right)^{+}\right.$ and $\left(\mu\left\llcorner\mathrm{e}+\sum_{\mathrm{v} \in \mathrm{e}} \tilde{a}_{\mathrm{e}, \mathrm{v}} \delta_{\mathrm{v}}\right)^{-}\right.$with respect to the cost given by the metric $d_{\Gamma}$. Then we have

$$
W_{1}\left(\left(\mu_{\mathrm{e}}+\sum_{\mathrm{v} \in \mathrm{e}}\left[\tilde{a}_{\mathrm{e}, \mathrm{v}} \delta_{\mathrm{v}}\right]_{\mathrm{e}}\right)^{+},\left(\mu_{\mathrm{e}}+\sum_{\mathrm{v} \in \mathrm{e}}\left[\tilde{a}_{\mathrm{e}, \mathrm{v}} \delta_{\mathrm{v}}\right]_{\mathrm{e}}\right)^{-}\right) \geq \int_{\Gamma \times \Gamma} d_{\Gamma}(x, y) d \gamma_{\mathrm{e}}(x, y) .
$$

Hence, if $\gamma:=\sum_{\mathrm{e} \in \mathrm{E}(\Gamma)} \gamma_{\mathrm{e}}$, we get

$$
\begin{aligned}
& \mathcal{C}_{\mu, a_{\mathrm{v}}}\left(\left\{\tilde{a}_{\mathrm{e}, \mathrm{v}}\right\}\right)=\sum_{\mathrm{e} \in \mathrm{E}(\Gamma)} W_{1}\left(\left(\mu_{\mathrm{e}}+\sum_{\mathrm{v} \in \mathrm{e}}\left[\tilde{a}_{\mathrm{e}, \mathrm{v}} \delta_{\mathrm{v}}\right]_{\mathrm{e}}\right)^{+},\left(\mu_{\mathrm{e}}+\sum_{\mathrm{v} \in \mathrm{e}}\left[\tilde{a}_{\mathrm{e}, \mathrm{v}} \delta_{\mathrm{v}}\right]_{\mathrm{e}}\right)^{-}\right) \\
& \geq \int_{\Gamma \times \Gamma} d_{\Gamma}(x, y) d \gamma(x, y) .
\end{aligned}
$$

Now, given $\varphi \in C(\Gamma)$, we have

$$
\begin{aligned}
\int_{\Gamma} \varphi & (x) d \pi_{1} \# \gamma(x)=\int_{\Gamma \times \Gamma} \varphi(x) d \gamma(x, y)=\sum_{\mathrm{e} \in \mathrm{E}(\Gamma)} \int_{\Gamma \times \Gamma} \varphi(x) d \gamma_{\mathrm{e}}(x, y) \\
= & \sum_{\mathrm{e} \in \mathrm{E}(\Gamma)} \int_{\Gamma} \varphi(x) d\left(\mu\left\llcorner\mathrm{e}+\sum_{\mathrm{v} \in \mathrm{e}} \tilde{a}_{\mathrm{e}, \mathrm{v}} \delta_{\mathrm{v}}\right)^{+}\right. \\
= & \sum_{\mathrm{e} \in \mathrm{E}(\Gamma)}\left(\int_{0}^{\ell_{\mathrm{e}}}[\varphi]_{\mathrm{e}}(x) d \mu_{\mathrm{e}}+\sum_{\mathrm{v} \in \mathrm{e}} \tilde{a}_{\mathrm{e}, \mathrm{v}}^{+} \varphi(\mathrm{v})\right) .
\end{aligned}
$$


Since

$$
\sum_{\mathrm{e} \in \mathrm{E}(\Gamma)} \sum_{\mathrm{v} \in \mathrm{e}} \tilde{a}_{\mathrm{e}, \mathrm{v}}^{+} \varphi(\mathrm{v})=\sum_{\mathrm{v} \in V(\Gamma)} a_{\mathrm{v}}^{+} \varphi(\mathrm{v})=\int_{\Gamma} \varphi(x) d\left(\sum_{\mathrm{v} \in \mathrm{V}(\Gamma)} a_{\mathrm{v}} \delta_{\mathrm{v}}\right)^{+}
$$

we get

$$
\pi_{1} \# \gamma=\left(\mu+\sum_{\mathrm{v} \in \mathrm{V}(\Gamma)} a_{\mathrm{v}} \delta_{\mathrm{v}}\right)^{+}
$$

Similarly, we obtain

$$
\pi_{2} \# \gamma=\left(\mu+\sum_{\mathrm{v} \in \mathrm{V}(\Gamma)} a_{\mathrm{v}} \delta_{\mathrm{v}}\right)^{-}
$$

Consequently,

$$
\gamma \in \prod\left(\left(\mu+\sum_{\mathrm{v} \in \mathrm{V}(\Gamma)} a_{\mathrm{v}} \delta_{\mathrm{v}}\right)^{+},\left(\mu+\sum_{\mathrm{v} \in \mathrm{V}(\Gamma)} a_{\mathrm{v}} \delta_{\mathrm{v}}\right)^{-}\right)
$$

Therefore,

$$
\begin{aligned}
\int_{\Gamma} u_{\infty}(\mu & \left.+\sum_{\mathrm{v} \in \mathrm{V}(\Gamma)} a_{\mathrm{v}} \delta_{\mathrm{v}}\right) \\
=\min & \left\{\int_{\Gamma \times \Gamma} d_{\Gamma}(x, y) d \sigma(x, y): \sigma \in \Pi\left(\eta^{+}, \eta^{-}\right)\right\} \\
\leq & \int_{\Gamma \times \Gamma} d_{\Gamma}(x, y) d \gamma(x, y) \leq \mathcal{C}_{\mu, a_{\mathrm{v}}}\left(\left\{\tilde{a}_{\mathrm{e}, \mathrm{v}}\right\}\right) \\
\leq & \mathcal{C}_{\mu, a_{\mathrm{v}}}\left(\left\{a_{\infty, \mathrm{e}, \mathrm{v}}\right\}\right) \\
& =\int_{\Gamma} u_{\infty}\left(\mu+\sum_{\mathrm{v} \in \mathrm{V}(\Gamma)} a_{\mathrm{v}} \delta_{\mathrm{v}}\right)
\end{aligned}
$$

from where we get (3.13) and (3.14).

Remark 3.7. Observe that (3.14) imply that each $a_{\infty, \mathrm{e}, \mathrm{v}}$ is the mass that enters/leaves e via the vertex $\mathrm{v} \in \mathrm{e}$, depending on its positive/negative sign, during the optimal transport process.

Remark 3.8. In the particular case of $\mu \equiv 0$ we get the even simpler formula

$$
\left\{a_{\infty, \mathrm{e}, \mathrm{v}}\right\}_{\substack{\mathrm{e} \in \mathrm{v} \in \mathrm{e}(\Gamma) \\ \arg \min }}\left\{\frac{1}{2} \sum_{\mathrm{e} \in \mathrm{E}(\Gamma)}\left(\sum_{\mathrm{v} \in \mathrm{e}}\left|a_{\mathrm{e}, \mathrm{v}}\right|\right) \ell_{\mathrm{e}}:\left\{a_{\mathrm{e}, \mathrm{v}}\right\} \text { solve }\left(L_{0, a_{\mathrm{v}}}\right)\right\}
$$

and

$$
W_{0, a_{\mathrm{v}}}=\min \left\{\frac{1}{2} \sum_{\mathrm{e} \in \mathrm{E}(\Gamma)}\left(\sum_{\mathrm{v} \in \mathrm{e}}\left|a_{\mathrm{e}, \mathrm{v}}\right|\right) \ell_{\mathrm{e}}:\left\{a_{\mathrm{e}, \mathrm{v}}\right\} \text { solve }\left(L_{0, a_{\mathrm{v}}}\right)\right\}
$$

for the optimal total cost of the transport problem. Observe that we can rewrite this as follows:

$$
W_{0, a_{\mathrm{v}}}=\min \left\{\sum_{\mathrm{e} \in \mathrm{E}(\Gamma)}\left|a_{\mathrm{e}, \mathrm{i}_{\mathrm{e}}}\right| \ell_{\mathrm{e}}:\left\{a_{\mathrm{e}, \mathrm{v}}\right\} \text { solve }\left(L_{0, a_{\mathrm{v}}}\right)\right\}
$$


Moreover, since

$$
a_{p, \mathrm{e}, \mathrm{i}_{\mathrm{e}}}+a_{p, \mathrm{e}, \mathrm{f}_{\mathrm{e}}}=0,
$$

we have $\left[u_{p}\right]_{\mathrm{e}}$ is a weak solution of

$$
\left\{\begin{array}{l}
-\left(\left|u^{\prime}\right|^{p-2} u^{\prime}\right)^{\prime}=0 \quad \text { in }\left(0, \ell_{\mathrm{e}}\right) \\
\left|\frac{\partial u}{\partial x_{\mathrm{e}}}\right|^{p-2} \frac{\partial u}{\partial x_{\mathrm{e}}}(0)=-a_{p, \mathrm{e}, \mathrm{i}_{\mathrm{e}}}, \\
\left|\frac{\partial u}{\partial x_{\mathrm{e}}}\right|^{p-2} \frac{\partial u}{\partial x_{\mathrm{e}}}\left(\ell_{\mathrm{e}}\right)=-a_{p, \mathrm{e}, \mathrm{i}_{\mathrm{e}}} .
\end{array}\right.
$$

Therefore, up to a constant,

$$
\left[u_{p}\right]_{\mathrm{e}}(x)=-\operatorname{sign}\left(a_{p, \mathrm{e}, \mathrm{i}_{\mathrm{e}}}\right)\left|a_{p, \mathrm{e}, \mathrm{i}_{\mathrm{e}}}\right|^{\frac{1}{p-1}} x,
$$

and consequently, up to a constant,

$$
\left[u_{\infty}\right]_{\mathrm{e}}(x)=-\operatorname{sign}\left(a_{\infty, \mathrm{e}, \mathrm{i}_{\mathrm{e}}}\right) x .
$$

A remarkable fact is that our results show that these functions can be glued continuously on the graph $\Gamma$.

In the next result we shall see that also in the case $\mu \neq 0$ it is possible to get a simple formula for the total cost. We also find a transport density for the transport problem.

Theorem 3.9. Let $\eta$ be a measure given by (1.1). Under the assumptions of Theorem 3.5 and for $\left\{a_{\infty, \mathrm{e}, \mathrm{v}}\right\}_{\substack{\mathrm{v} \in \mathrm{E}(\Gamma) \\ \mathrm{v}}}$ given there, there exists a nonnegative function $\mathbf{a} \in L^{\infty}(\Gamma)$ such that $\left[u_{\infty}\right]_{\mathrm{e}}$ is a weak solution of

$$
\left\{\begin{array}{l}
-\left([\mathbf{a}]_{\mathrm{e}}\left[u_{\infty}\right]_{\mathrm{e}}^{\prime}\right)^{\prime}=\mu_{\mathrm{e}} \quad \text { in }\left(0, \ell_{\mathrm{e}}\right), \\
{[\mathbf{a}]_{\mathrm{e}}\left[u_{\infty}\right]_{\mathrm{e}}^{\prime}(0)=-a_{\infty, \mathrm{e}, \mathrm{i}_{\mathrm{e}}},} \\
{[\mathbf{a}]_{\mathrm{e}}\left[u_{\infty}\right]_{\mathrm{e}}^{\prime}\left(\ell_{\mathrm{e}}\right)=a_{\infty, \mathrm{e}, \mathrm{f}_{\mathrm{e}}},}
\end{array}\right.
$$

in the sense

$$
\int_{0}^{\ell_{\mathrm{e}}}[\mathbf{a}]_{\mathrm{e}}\left[u_{\infty}\right]_{\mathrm{e}}^{\prime} \varphi^{\prime}-\int_{0}^{\ell_{\mathrm{e}}} \varphi d \mu_{\mathrm{e}}=a_{\infty, \mathrm{e}, \mathrm{f}_{\mathrm{e}}} \varphi\left(\ell_{\mathrm{e}}\right)+a_{\infty, \mathrm{e}, \mathrm{i}_{\mathrm{e}}} \varphi(0)
$$

for all $\varphi \in W^{1, \infty}\left(0, \ell_{\mathrm{e}}\right)$. Furthermore,

$$
\left|\left[u_{\infty}\right]_{\mathrm{e}}^{\prime}\right|=1 \quad \text { on }\left\{[\mathbf{a}]_{\mathrm{e}}>0\right\} .
$$

More precisely, we have

$$
[\mathbf{a}]_{\mathrm{e}}(x)=\left|a_{\infty, \mathrm{e}, \mathrm{i}_{\mathrm{e}}}+\int_{0}^{x} d \mu_{\mathrm{e}}(y)\right| \quad \text { for } x \in\left(0, \ell_{\mathrm{e}}\right)
$$

and

$$
\left[u_{\infty}\right]_{\mathrm{e}}^{\prime}(x)=\operatorname{sign}\left(-a_{\infty, \mathrm{e}, \mathrm{i}_{\mathrm{e}}}-\int_{0}^{x} d \mu_{\mathrm{e}}(y)\right) \quad \text { for } x \in\left(0, \ell_{\mathrm{e}}\right) .
$$


Moreover, it holds that the optimal total cost is given by

$$
\begin{aligned}
W_{\mu, a_{\mathrm{v}}}= & \min \left\{\sum_{\mathrm{e} \in \mathrm{E}(\Gamma)} \int_{0}^{\ell \mathrm{e}}\left|a_{\mathrm{e}, \mathrm{i}_{\mathrm{e}}}+\int_{0}^{x} d \mu_{\mathrm{e}}(y)\right| d x:\left\{a_{\mathrm{e}, \mathrm{v}}\right\} \text { solve }\left(L_{\mu, a_{\mathrm{v}}}\right)\right\} \\
& =\sum_{\mathrm{e} \in \mathrm{E}(\Gamma)} \int_{0}^{\ell \mathrm{e}}\left|a_{\infty, \mathrm{e}, \mathrm{i}_{\mathrm{e}}}+\int_{0}^{x} d \mu_{\mathrm{e}}(y)\right| d x .
\end{aligned}
$$

Proof. Fix e $\in E(\Gamma)$, and take $\left[u_{p}\right]_{\mathrm{e}}$ the weak solution obtained above. Then, from (3.10) we have

$$
-\left(\left|\left[u_{p}\right]_{\mathrm{e}}^{\prime}\right|^{p-2}\left[u_{p}\right]_{\mathrm{e}}^{\prime}\right)^{\prime}=\mu_{\mathrm{e}} \quad \text { in } \mathcal{D}^{\prime}\left(0, \ell_{\mathrm{e}}\right)
$$

Now, we let

$$
w_{\mu, \mathrm{e}}(x):=\mu_{\mathrm{e}}((0, x))=\int_{0}^{x} d \mu_{\mathrm{e}}(y)
$$

for $x \in\left(0, \ell_{\mathrm{e}}\right)$ and we observe that $\left(w_{\mu, \mathrm{e}}\right)^{\prime}=\mu_{\mathrm{e}}$ in $\mathcal{D}^{\prime}\left(0, \ell_{\mathrm{e}}\right)$. Hence, there exists a constant $\alpha_{p, \mathrm{e}} \in \mathbb{R}$ such that $\left|\left[u_{p}\right]_{\mathrm{e}}^{\prime}\right|^{p-2}\left[u_{p}\right]_{\mathrm{e}}^{\prime}=-w_{\mu, \mathrm{e}}+\alpha_{p, \mathrm{e}}$. Then, since

$$
-a_{p, \mathrm{e}, \mathrm{i}_{\mathrm{e}}}=\left|\left[u_{p}\right]_{\mathrm{e}}^{\prime}\right|^{p-2}\left[u_{p}\right]_{\mathrm{e}}^{\prime}(0)=-w_{\mu, \mathrm{e}}(0)+\alpha_{p, \mathrm{e}}=\alpha_{p, \mathrm{e}},
$$

we get

$$
\left|\left[u_{p}\right]_{\mathrm{e}}^{\prime}\right|^{p-2}\left[u_{p}\right]_{\mathrm{e}}^{\prime}(x)=-w_{\mu, \mathrm{e}}(x)-a_{p, \mathrm{e}, \mathrm{i}_{\mathrm{e}}} .
$$

Now we observe that $-w_{\mu, \mathrm{e}}(x)-a_{p, \mathrm{e}, \mathrm{i}_{\mathrm{e}}}$ is bounded in $L^{\infty}$ uniformly in $p$, and that, for a subsequence,

$\left|\left[u_{p}\right]_{\mathrm{e}}^{\prime}(x)\right|^{p-2}=\left|w_{\mu, \mathrm{e}}(x)+a_{p, \mathrm{e}, \mathrm{i}_{\mathrm{e}}}\right|^{\frac{p-2}{p-1}} \rightarrow\left|w_{\mu, \mathrm{e}}(x)+a_{\infty, \mathrm{e}, \mathrm{i}_{\mathrm{e}}}\right|=:[\mathbf{a}]_{\mathrm{e}}(x) \quad \forall x \in\left(0, \ell_{\mathrm{e}}\right)$.

Moreover, by (3.12), we can assume that

$$
\left[u_{p}\right]_{\mathrm{e}}^{\prime} \rightarrow\left[u_{\infty}\right]_{\mathrm{e}}^{\prime} \quad \text { weakly in } L^{2}\left(\left(0, \ell_{\mathrm{e}}\right)\right) .
$$

Therefore, we have that

$$
\left|\left[u_{p}\right]_{\mathrm{e}}^{\prime}\right|^{p-2}\left[u_{p}\right]_{\mathrm{e}}^{\prime} \rightarrow\left|w_{\mu, \mathrm{e}}+a_{\infty, \mathrm{e}, \mathrm{i}_{\mathrm{e}}}\right|\left[u_{\infty}\right]_{\mathrm{e}}^{\prime} \quad \text { in } L^{1}\left(\left(0, \ell_{\mathrm{e}}\right)\right),
$$

but also

$$
\left|\left[u_{p}\right]_{\mathrm{e}}^{\prime}\right|^{p-2}\left[u_{p}\right]_{\mathrm{e}}^{\prime} \rightarrow-w_{\mu, \mathrm{e}}-a_{\infty, \mathrm{e}, \mathrm{i}_{\mathrm{e}}} .
$$

Consequently, (3.16), (3.17) and (3.18) are proved.

On the other hand, by $(3.11)$, for any $\varphi \in W^{1, \infty}\left(0, \ell_{\mathrm{e}}\right)$, we have

$$
\int_{0}^{\ell_{\mathrm{e}}}\left|\left[u_{p}\right]_{\mathrm{e}}^{\prime}\right|^{p-2}\left[u_{p}\right]_{\mathrm{e}}^{\prime} \varphi^{\prime}-\int_{0}^{\ell_{\mathrm{e}}} \varphi d \mu_{\mathrm{e}}=a_{p, \mathrm{e}, \mathrm{f}_{\mathrm{e}}} \varphi\left(\ell_{\mathrm{e}}\right)+a_{p, \mathrm{e}, \mathrm{i}_{\mathrm{e}}} \varphi(0) .
$$

Then, taking limits in (3.20) when $p \rightarrow \infty$, we get

$$
\int_{0}^{\ell_{\mathrm{e}}}[\mathbf{a}]_{\mathrm{e}}\left[u_{\infty}\right]_{\mathrm{e}}^{\prime} \varphi^{\prime}-\int_{0}^{\ell_{\mathrm{e}}} \varphi d \mu_{\mathrm{e}}=a_{\infty, \mathrm{e}, \mathrm{f}_{\mathrm{e}}} \varphi\left(\ell_{\mathrm{e}}\right)+a_{\infty, \mathrm{e}, \mathrm{i}_{\mathrm{e}}} \varphi(0)
$$

for all $\varphi \in W^{1, \infty}\left(0, \ell_{\mathrm{e}}\right)$, and thus (3.15) holds.

Let $\left\{a_{\mathrm{e}, \mathrm{v}}\right\}$ a solution of $\left(L_{\mu, a_{\mathrm{v}}}\right)$. Then, if we define

$$
\tilde{\mathbf{a}}(x)=\left|a_{e, \mathrm{i}_{\mathrm{e}}}+\int_{0}^{x} d \mu_{\mathrm{e}}(y)\right| \quad \text { for } x \in\left(0, \ell_{\mathrm{e}}\right),
$$

and $\tilde{u}$ by

$$
\tilde{u}^{\prime}(x)=\operatorname{sign}\left(-a_{e, \mathrm{i}_{\mathrm{e}}}-\int_{0}^{x} d \mu_{\mathrm{e}}(y)\right) \text { for } x \in\left(0, \ell_{\mathrm{e}}\right),
$$


we have, $0 \leq \tilde{\mathbf{a}} \in L^{\infty}\left(0, \ell_{\mathrm{e}}\right)$ and $\tilde{u}$ is a Lipschitz function with $\left\|\tilde{u}^{\prime}\right\|_{L^{\infty}\left(0, \ell_{\mathrm{e}}\right)} \leq 1$, such that $\tilde{u}$ is a weak solution of

$$
\left\{\begin{array}{l}
-\left(\tilde{\mathbf{a}} \tilde{u}^{\prime}\right)^{\prime}=\mu_{\mathrm{e}} \quad \text { in }\left(0, \ell_{\mathrm{e}}\right), \\
\tilde{\mathbf{a}} \tilde{u}^{\prime}(0)=-a_{\mathrm{e}, \mathrm{i}_{\mathrm{e}}} \\
\tilde{\mathbf{a}} \tilde{u}^{\prime}\left(\ell_{\mathrm{e}}\right)=a_{\mathrm{e}, \mathrm{f}_{\mathrm{e}}}
\end{array}\right.
$$

and

$$
\left|\tilde{u}^{\prime}\right|=1 \quad \text { on }\{\tilde{\mathbf{a}}>0\} .
$$

Then by (ii) in Lemma 3.4, $\tilde{u}$ is a Kantorovich potential for the transport of $\left(\mu_{\mathrm{e}}+\sum_{\mathrm{v} \in \mathrm{e}}\left[a_{\mathrm{e}, \mathrm{v}} \delta_{\mathrm{v}}\right]_{\mathrm{e}}\right)^{+}$to $\left(\mu_{\mathrm{e}}+\sum_{\mathrm{v} \in \mathrm{e}}\left[a_{\mathrm{e}, \mathrm{v}} \delta_{\mathrm{v}}\right]_{\mathrm{e}}\right)^{-}$. Therefore, taking $\tilde{u}$ as test function in (3.21), we get

$$
\int_{0}^{\ell_{\mathrm{e}}} \tilde{\mathbf{a}}\left|\tilde{u}^{\prime}\right|^{2}-\int_{0}^{\ell_{\mathrm{e}}} \tilde{u} d \mu_{\mathrm{e}}=a_{\mathrm{e}, \mathrm{f}_{\mathrm{e}}} \tilde{u}\left(\ell_{\mathrm{e}}\right)+a_{\mathrm{e}, \mathrm{i}_{\mathrm{e}}} \tilde{u}(0),
$$

and consequently

$$
\begin{gathered}
\int_{0}^{\ell_{\mathrm{e}}}\left|a_{e, \mathrm{i}_{\mathrm{e}}}+\int_{0}^{x} d \mu_{\mathrm{e}}(y)\right| d x=-\int_{0}^{\ell_{\mathrm{e}}} \tilde{u} d \mu_{\mathrm{e}}+a_{\mathrm{e}, \mathrm{f}_{\mathrm{e}}} \tilde{u}\left(\ell_{\mathrm{e}}\right)+a_{\mathrm{e}, \mathrm{i}_{\mathrm{e}}} \tilde{u}(0) \\
=W_{1}\left(\left(\mu_{\mathrm{e}}+\sum_{\mathrm{v} \in \mathrm{e}}\left[a_{\mathrm{e}, \mathrm{v}} \delta_{\mathrm{v}}\right]_{\mathrm{e}}\right)^{+},\left(\mu_{\mathrm{e}}+\sum_{\mathrm{v} \in \mathrm{e}}\left[a_{\mathrm{e}, \mathrm{v}} \delta_{\mathrm{v}}\right]_{\mathrm{e}}\right)^{-}\right) .
\end{gathered}
$$

Adding, we obtain

$$
\sum_{\mathrm{e} \in \mathrm{E}(\Gamma)} \int_{0}^{\ell \mathrm{e}}\left|a_{\mathrm{e}, \mathrm{i}_{\mathrm{e}}}+\int_{0}^{x} d \mu_{\mathrm{e}}(y)\right| d x=\mathcal{C}_{\mu, a_{\mathrm{v}}}\left(\left\{a_{\mathrm{e}, \mathrm{v}}\right\}\right),
$$

from where (3.19) follows having in mind (3.14).

Remark 3.10. The first part of the above result is similar to Lemma 7.2 in [1] the main difference is that here we have to consider fluxes at the ends of the edges.

Finally, let us present some simple examples to illustrate our results.

Example 3.11. Let $\Gamma$ be the metric graph with edges $E(\Gamma)=\left\{\mathrm{e}_{1}, \mathrm{e}_{2}, \mathrm{e}_{3}, \mathrm{e}_{4}\right\}$ and vertices $V(\Gamma)=\left\{\mathrm{v}_{1}, \mathrm{v}_{2}, \mathrm{v}_{3}, \mathrm{v}_{4}\right\}$ such that $\left(\mathrm{i}_{\mathrm{e}_{1}}, \mathrm{f}_{\mathrm{e}_{1}}\right)=\left(\mathrm{v}_{1}, \mathrm{v}_{2}\right),\left(\mathrm{i}_{\mathrm{e}_{2}}, \mathrm{f}_{\mathrm{e}_{2}}\right)=\left(\mathrm{v}_{2}, \mathrm{v}_{3}\right)$, $\left(\mathrm{i}_{\mathrm{e}_{3}}, \mathrm{f}_{\mathrm{e}_{3}}\right)=\left(\mathrm{v}_{2}, \mathrm{v}_{4}\right),\left(\mathrm{i}_{\mathrm{e}_{4}}, \mathrm{f}_{\mathrm{e}_{4}}\right)=\left(\mathrm{v}_{3}, \mathrm{v}_{4}\right)$ (see FIGURE 1$)$.

Consider the measure

$$
\eta=\mu+\sum_{\mathrm{v} \in V(\Gamma)} a_{\mathrm{v}} \delta_{\mathrm{v}}
$$

with $\mu_{\mathrm{e}_{1}}^{+}=\chi_{\left(0, \frac{\ell_{1}}{2}\right)}$, all the other $\mu_{\mathrm{e}_{i}}^{ \pm}=0$, and $a_{\mathrm{v}_{1}}=a_{\mathrm{v}_{2}}=0, a_{\mathrm{v}_{3}}=a_{\mathrm{v}_{4}}=-\frac{\ell_{\mathrm{e}_{1}}}{4}$. By Theorem 1.2, we have that

$$
\begin{array}{r}
W_{\mu, a_{\mathrm{v}}}=\min _{\left\{a_{i j}\right\} \text { solve }\left(L_{\mu, a_{\mathrm{v}}}\right)}\left(\int_{0}^{\frac{l_{1}}{2}}\left|a_{11}+x\right| d x+\int_{\frac{l_{1}}{2}}^{l_{1}}\left|a_{11}+\frac{l_{1}}{2}\right| d x\right. \\
\left.+\left|a_{22}\right| l_{2}+\left|a_{32}\right| l_{3}+\left|a_{43}\right| l_{4}\right),
\end{array}
$$

where $a_{i j}=a_{\mathrm{e}_{i}, \mathrm{v}_{j}}$ and $l_{i}=\ell_{\mathrm{e}_{i}}$. Now, a simple calculation shows that

$$
a_{11}=0, \quad a_{12}=-\frac{l_{1}}{2}, \quad a_{23}=-a_{22}, \quad a_{32}=\frac{l_{1}}{2}-a_{22},
$$


and

Hence, the total cost is given by

$$
a_{34}=a_{22}-\frac{l_{1}}{2}, \quad a_{43}=a_{22}-\frac{l_{1}}{4}, \quad a_{44}=\frac{l_{1}}{4}-a_{22} .
$$

$$
W_{\mu, a_{\mathrm{v}}}=\min _{a_{22} \in \mathbb{R}}\left(\frac{3}{8} l_{1}^{2}+\left|a_{22}\right| l_{2}+\left|a_{22}-\frac{l_{1}}{2}\right| l_{3}+\left|a_{22}-\frac{l_{1}}{4}\right| l_{4}\right) .
$$

We have different values for the minimum depending on the values of $l_{2}, l_{3}, l_{4}$ :

(1) If $l_{2}>l_{3}+l_{4}$ then the minimum is attained at $a_{22}=0$. Consequently, as expected, the route of the optimal transport is given in FigurE 1. Note that we are not sending mass trough $e_{2}$, but instead we use $e_{3}$ until we reach $v_{4}$ where we deposit half of the mass and then continue trough $\mathrm{e}_{4}$ until reaching $\mathrm{v}_{3}$ with the other half of the mass.

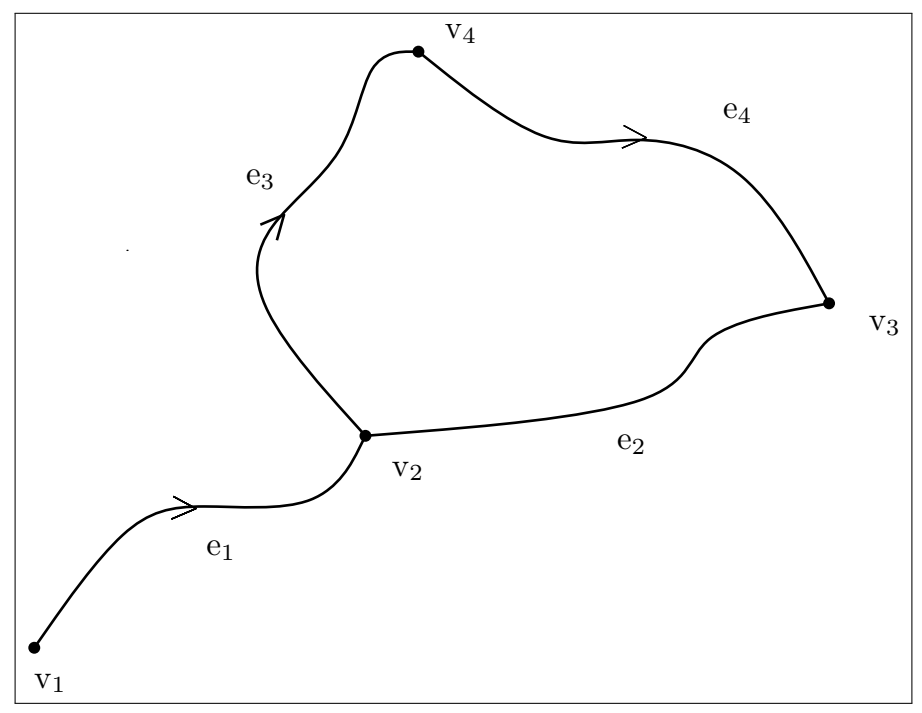

Figure 1. Optimal transport path for $l_{2}>l_{3}+l_{4}$

In this case, the optimal transport cost is given by

$$
W_{\mu, a_{\mathrm{v}}}=\frac{3}{8} l_{1}^{2}+\frac{1}{2} l_{1} l_{2}+\frac{1}{4} l_{1} l_{4}=\frac{1}{2} l_{1} \frac{3}{4} l_{1}+\frac{1}{4} l_{1} l_{3}+\frac{1}{4} l_{1}\left(l_{3}+l_{4}\right) .
$$

(2) If $l_{3}>l_{2}+l_{4}$ then the minimum is attained at $a_{22}=\frac{l_{1}}{2}$. Hence $a_{32}=0$ and consequently, in this case, the best strategy is not to use $e_{3}$ to transport mass.

(3) In other case, the minimum is attained at $a_{22}=\frac{l_{1}}{4}$. Hence $a_{43}=a_{44}=0$, therefore, here we split the mass in two equal parts when we arrive to $\mathrm{v}_{2}$ and send them to $\mathrm{v}_{3}$ and $\mathrm{v}_{4}$ using $\mathrm{e}_{2}$ and $\mathrm{e}_{3}$. Now, we are not using $\mathrm{e}_{4}$.

Example 3.12. Let $\Gamma$ be the metric graph with edges $E(\Gamma)=\left\{\mathrm{e}_{1}, \mathrm{e}_{2}, \mathrm{e}_{3}\right\}$ and vertices $V(\Gamma)=\left\{\mathrm{v}_{1}, \mathrm{v}_{2}, \mathrm{v}_{3}\right\}$ such that $\left(\mathrm{i}_{\mathrm{e}_{1}}, \mathrm{f}_{\mathrm{e}_{1}}\right)=\left(\mathrm{v}_{1}, \mathrm{v}_{2}\right),\left(\mathrm{i}_{\mathrm{e}_{2}}, \mathrm{f}_{\mathrm{e}_{2}}\right)=\left(\mathrm{v}_{2}, \mathrm{v}_{3}\right)$, $\left(\mathrm{i}_{\mathrm{e}_{3}}, \mathrm{f}_{\mathrm{e}_{3}}\right)=\left(\mathrm{v}_{3}, \mathrm{v}_{1}\right)$ (see FIGURE 2$)$. Take $\ell_{\mathrm{e}_{1}}=1$.

Consider the measure

$$
\eta=\mu+\sum_{\mathrm{v} \in V(\Gamma)} a_{\mathrm{v}} \delta_{\mathrm{v}}
$$


with $\mu_{\mathrm{e}_{1}}^{+}=\chi_{(0, \alpha)}, 0<\alpha \leq 1$, all the other $\mu_{\mathrm{e}_{i}}^{ \pm}=0$, and $a_{\mathrm{v}_{1}}=a_{\mathrm{v}_{2}}=a_{\mathrm{v}_{3}}=-\frac{\alpha}{3}$. By Theorem 1.2, we have that

$$
\begin{aligned}
W_{\mu, a_{\mathrm{v}}}=\min _{\left\{a_{i j}\right\} \text { solve }\left(L_{\mu, a_{\mathrm{v}}}\right)}( & \int_{0}^{\alpha}\left|a_{11}+x\right| d x \\
& \left.+\left|a_{11}+\alpha\right|(1-\alpha)+\left|a_{22}\right| l_{2}+\left|a_{31}\right| l_{3}\right),
\end{aligned}
$$

where $a_{i j}=a_{\mathrm{e}_{i}, \mathrm{v}_{j}}$ and $l_{i}=\ell_{\mathrm{e}_{i}}$. Now, a simple calculation shows that

$a_{12}=-a_{11}-\alpha, \quad a_{22}=a_{11}+\frac{2}{3} \alpha, \quad a_{23}=-a_{22}, \quad a_{31}=-a_{11}-\frac{1}{3} \alpha, \quad a_{33}=-a_{31}$.

Therefore,

$$
\begin{array}{r}
W_{\mu, a_{\mathrm{v}}}=\min _{a_{11} \in \mathbb{R}}\left(\int_{0}^{\alpha}\left|a_{11}+x\right| d x+\left|a_{11}+\alpha\right|(1-\alpha)\right. \\
\left.+\left|a_{11}+\frac{2}{3} \alpha\right| l_{2}+\left|a_{11}+\frac{1}{3} \alpha\right| l_{3}\right) .
\end{array}
$$

We have different values for the minimum depending on the values of $l_{2}, l_{3}$ and $\alpha$. In fact, preforming a tedious computation we get the following:

(1) If $\alpha \geq \frac{3}{2}\left(1+l_{2}-l_{3}\right)$ then the minimum is attained at $a_{11}=-\frac{1}{3} \alpha$. This implies that

$$
a_{12}=-\frac{2}{3} \alpha, \quad a_{22}=\frac{1}{3} \alpha, \quad a_{23}=-\frac{1}{3} \alpha, \quad a_{31}=a_{33}=0 .
$$

The path of the optimal transport is given in FigurE 2 (a).

Note that we are not sending mass trough $e_{3}$, but instead we use $e_{1}$ until we reach $\mathrm{v}_{2}$ where we deposit $\frac{1}{3} \alpha$ of the mass and then continue trough $\mathrm{e}_{2}$ until reaching $\mathrm{v}_{3}$ with the other $\frac{1}{3} \alpha$ of the mass.

(2) If $\frac{3}{4}\left(1+l_{2}-l_{3}\right)<\alpha<\frac{3}{2}\left(1+l_{2}-l_{3}\right)$ then the minimum is attained at $a_{11}=$ $\frac{1}{2}\left(l_{3}-l_{2}-1\right) \in\left(-\frac{2}{3} \alpha,-\frac{1}{3} \alpha\right)$. Therefore, all the $a_{i j} \neq 0$ and consequently in this case it is necessary to use all the edges.

(3) If $\frac{3}{4}\left(1-l_{2}-l_{3}\right) \leq \alpha \leq \frac{3}{4}\left(1+l_{2}-l_{3}\right)$ then the minimum is attained at $a_{11}=-\frac{2}{3} \alpha$. Hence,

$$
a_{12}=-\frac{1}{3} \alpha, \quad a_{22}=a_{23}=0, \quad a_{31}=-\frac{1}{3} \alpha \quad a_{33}=\frac{1}{3} \alpha .
$$

Consequently, in this case, the best strategy is not to use $\mathrm{e}_{2}$ to transport mass.

(4) If $\frac{1}{2}\left(1-l_{2}-l_{3}\right)<\alpha<\frac{3}{4}\left(1-l_{2}-l_{3}\right)$ then the minimum is attained at $a_{11}=$ $\frac{1}{2}\left(l_{3}+l_{2}-1\right) \in\left(-\alpha,-\frac{2}{3} \alpha\right)$. In this case, the best strategy is not to use $\mathrm{e}_{3}$ to transport mass.

(5) If $\alpha \leq \frac{1}{2}\left(1-l_{2}-l_{3}\right)$ then the minimum is attained at $a_{11}=-\alpha$. Then, we get

$$
a_{12}=0, \quad a_{22}=-\frac{1}{3} \alpha, \quad a_{23}=\frac{1}{3} \alpha, \quad a_{31}=\frac{2}{3} \alpha, \quad a_{33}=-\frac{2}{3} .
$$

In this case we are sending all the mass trough $\mathrm{v}_{1}$ and then use $\mathrm{e}_{3}$ and $\mathrm{e}_{2}$ to deliver it to its final destination at $\mathrm{v}_{3}$ and $\mathrm{v}_{2}$ ( see FIGURE $2(\mathrm{~b})$ ). 

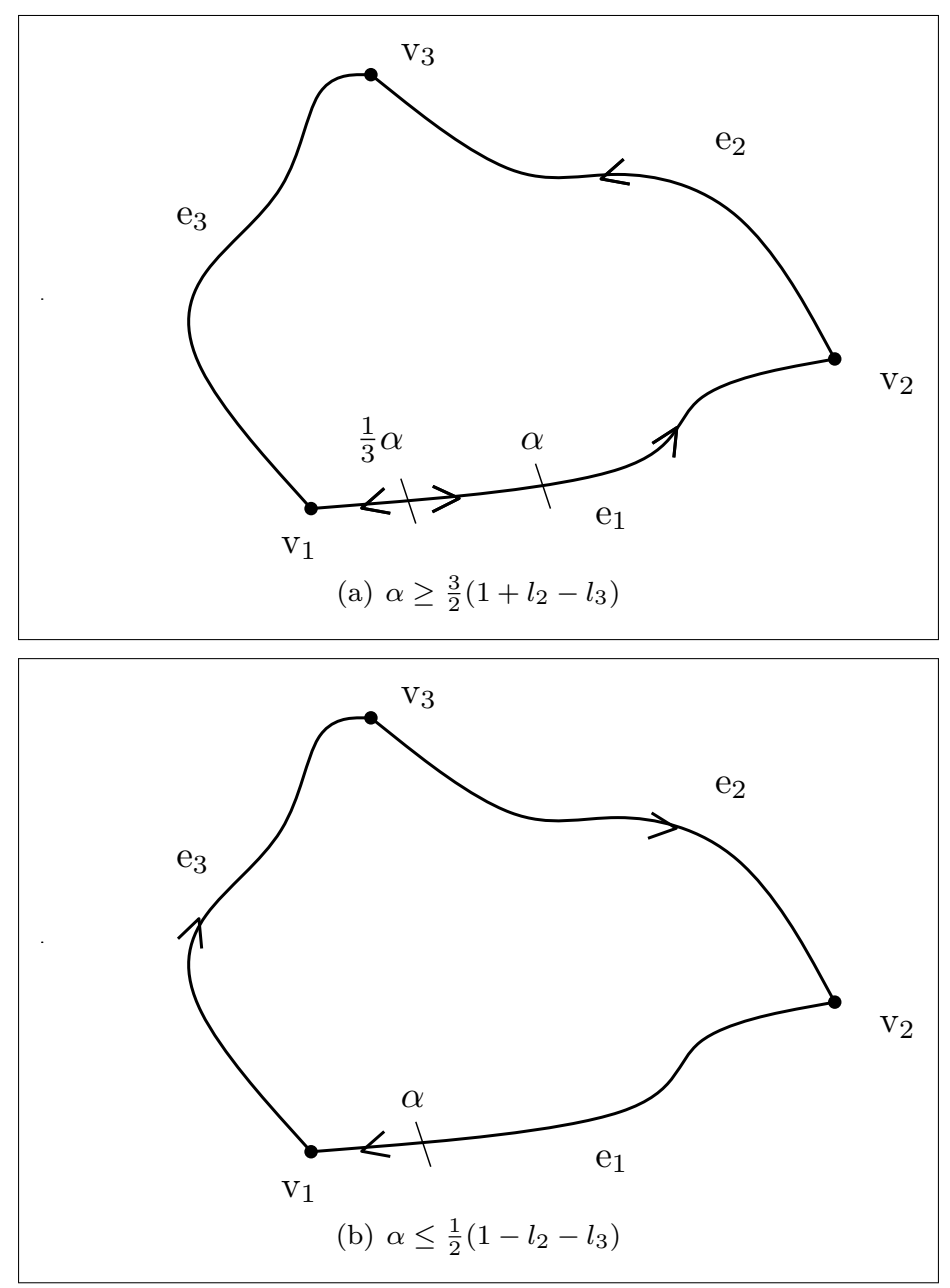

FiguRE 2. Optimal transport paths for different $\alpha$

\section{REFERENCES}

1. L. Ambrosio. Lecture notes on optimal transport problems. Mathematical aspects of evolving interfaces (Funchal, 2000), 1-52, Lecture Notes in Math., 1812, Springer, Berlin, 2003.

2. V. Banica and L. I. Ignat. Dispersion for the Schrödinger equation on networks. J. Math. Phys. 52 (2011), no. 8, 083703, 14 pp.

3. G. Berkolaiko and P. Kuchment. Introduction to quantum graphs. Mathematical Surveys and Monographs, 186. American Mathematical Society, Providence, RI, 2013. xiv+270 pp.

4. M. Bernot, V. Caselles and J. M. Morel. Optimal Transportation Networks. Lecture Notes in Math., 1955, Springer, Berlin, 2009.

5. G. Buttazzo, A. Pratelli, S. Solimini and E. Stepanov, Optimal urban networks via mass transportation. Lecture Notes inMathematics, 1961. Springer-Verlag, Berlin, 2009.

6. L. C. Evans. Partial differential equations. Graduate Studies in Mathematic, vol 19 Amer. Math. Soc. 1998.

7. L. C. Evans and W. Gangbo, Differential equations methods for the Monge-Kantorovich mass transfer problem. Mem. Amer. Math. Soc., 137 (1999), no. 653.

8. N. Igbida, J. M. Mazón, J. D. Rossi and J. Toledo. A Monge-Kantorovich mass transport problem for a discrete distance. J. Funct. Anal. 260, (2011), 3494-3534. 
9. V. Kostrykin, R. Schrader, Kirchhoff's Rule for Quantum Wires. J. Phys. A 32 (1999), no. 4, 595-630.

10. P. Kuchment, Quantum graphs: I. Some basic structures, Waves Random Media 14 (2004), S107-S128.

11. J. M. Mazón, J. D. Rossi and J. Toledo. An optimal transportation problem with a cost given by the Euclidean distance plus import/export taxes on the boundary. Revista Matemática Iberoamericana. 30 (2014), 277-308.

12. J. M. Mazón, J. D. Rossi and J. Toledo. An optimal matching problem for the Euclidean distance. SIAM J. Math. Anal. 46, (2014), 233-255.

13. J. M. Mazón, J. D. Rossi and J. Toledo. Mass transport problems for the Euclidean distance obtained as limits of p-Laplacian type problems with obstacles. J. Diff. Equations, 256 (2014), $3208-3244$.

14. J. M. Mazón, J. D. Rossi and J. Toledo. Mass transport problems obtained as limits of p-Laplacian type problems with spatial dependence. Adv. Non. Anal. 3(3), (2014), 133-140.

15. O. Post, Spectral Analysis on Graph-Like Spaces. Lecture Notes in Mathematics. 2012.

16. C. Villani. Topics in Optimal Transportation. Graduate Studies in Mathematics. Vol. 58, 2003.

17. C. Villani. Optimal transport. Old and new. Grundlehren der MathematischenWissenschaften (Fundamental Principles of Mathematical Sciences), vol. 338. Springer, Berlin (2009).

J. M. Mazón: Departament d’Anàlisi Matemàtica, Universitat de València, Valencia, SpaIN. mazon@uv.es

J. D. Rossi: COniCet and Depto de Matemática, FCEyn, Universidad de Buenos Aires, Pab. I, Ciudad Universitaria (1428), Buenos Aires, Argentina. jrossi@dm.uba.ar

J. Toledo: Departament d’Anàlisi Matemàtica, Universitat de València, València, SPAIN. toledojj@uv.es 